TRANSACTIONS OF THE

AMERICAN MATHEMATICAL SOCIETY

Volume 361, Number 9, September 2009, Pages 4969-4997

S 0002-9947(09)04796-5

Article electronically published on April 21, 2009

\title{
INTERSECTION NUMBERS OF POLYGON SPACES
}

\author{
JOSÉ AGAPITO AND LEONOR GODINHO
}

\begin{abstract}
We study the intersection ring of the space $\mathcal{M}\left(\alpha_{1}, \ldots, \alpha_{m}\right)$ of polygons in $\mathbb{R}^{3}$. We find homology cycles dual to generators of this ring and prove a recursion relation in $m$ (the number of edges) for their intersection numbers. This result is an analog of the recursion relation appearing in the work of Witten and Kontsevich on moduli spaces of punctured curves and in the work of Weitsman on moduli spaces of flat connections on two-manifolds of genus $g$ with $m$ marked points. Based on this recursion formula we obtain an explicit expression for the computation of the intersection numbers of polygon spaces and use it in several examples. Among others, we study the special case of equilateral polygon spaces (where all $\alpha_{i}$ 's are the same) and compare our results with the expressions for these particular spaces that have been determined by Kamiyama and Tezuka. Finally, we relate our explicit formula for the intersection numbers with the generating function for intersection pairings of the moduli space of flat connections of Yoshida, as well as with equivalent expressions for polygon spaces obtained by Takakura and Konno through different techniques.
\end{abstract}

\section{INTRODUCTION}

A Polygon space $\mathcal{M}(\alpha):=\mathcal{M}\left(\alpha_{1}, \ldots, \alpha_{m}\right), \alpha_{i} \in \mathbb{R}_{+}$, is the set of all configurations of closed piecewise linear paths in $\mathbb{R}^{3}$ with $m$ steps of lengths $\alpha_{1}, \ldots, \alpha_{m}$, modulo rotations and translations. These spaces have been widely studied in recent years. For example, Hausmann and Knutson computed their integer cohomology rings in the (generic) case that they are smooth 8]. Previously Klyachko [13 had showed that the cohomology groups were torsion free and calculated their rank. Moreover, Brion [1] and Kirwan [12 computed the rational cohomology ring for the particular case of equilateral polygons (that is, when the $\alpha_{i}$ 's are all equal) with an odd number of edges as well as this equilateral case modulo the symmetric group. This quotient of the equal-length space by the symmetric group is particularly interesting since it is a compactification of the moduli space of $m$-times-punctured Riemann spheres as well as a compactification of the moduli space of $m$ unordered points in $\mathbb{C} P^{1}$.

Let us state our main results. In Section 3 we associate a circle bundle $V_{i}$ on $\mathcal{M}(\alpha)$ to each edge $i=1, \ldots, m$ obtaining a list of degree-2 classes: their first Chern classes $c_{i}:=c_{1}\left(V_{i}\right)$. It is shown in $\left[8\right.$, that these classes generate $H^{*}\left(\mathcal{M}(\alpha) ; \mathbb{Z}\left[\frac{1}{2}\right]\right)$.

Received by the editors November 2, 2007.

2000 Mathematics Subject Classification. Primary 53D20, 58D99; Secondary 53D35.

The first author was partially supported by FCT (Portugal) through program POCTI/FEDER and grant POCTI/SFRH/BPD/20002/2004.

The second author was partially supported by FCT through program POCTI/FEDER and grant POCTI/MAT/57888/2004, and by Fundação Calouste Gulbenkian.

(C)2009 American Mathematical Society Reverts to public domain 28 years from publication 
The purpose of our work is the study of their intersection numbers, obtaining a recursion relation in $m$ as well as an explicit formula for their computation and examining its relation with other existing formulas by Konno [14, Takakura [20], Kamiyama and Tezuka [17] and by Yoshida 27] (this one in the context of moduli spaces of flat $S U(2)$-connections on the $m$-punctured sphere).

As we will see in Section 4 there exist natural homology cycles dual to these Chern classes which will allow us to perform computations in the cohomology ring via intersection theory. In particular, we develop a recursion relation in $m$, the number of edges. For that we start by showing that, for $\alpha_{j} \neq \alpha_{i}$, the homology class dual to $c_{i}$ is given by

$$
\left[D_{i, j}^{+}(\alpha)\right]+\operatorname{sgn}\left(\alpha_{i}-\alpha_{j}\right)\left[D_{i, j}^{-}(\alpha)\right]
$$

where $D_{i, j}^{+}(\alpha)$ and $D_{i, j}^{-}(\alpha)$ are codimension-2 submanifolds of $\mathcal{M}(\alpha)$ symplectomorphic to polygon spaces $\mathcal{M}\left(\alpha_{i, j}^{+}\right)$and $\mathcal{M}\left(\alpha_{i, j}^{-}\right)$with

$$
\begin{aligned}
& \alpha_{i, j}^{+}:=\left(\alpha_{1}, \ldots, \hat{\alpha}_{i}, \ldots, \hat{\alpha}_{j}, \ldots, \alpha_{i}+\alpha_{j}\right), \\
& \alpha_{i, j}^{-}:=\left(\alpha_{1}, \ldots, \hat{\alpha}_{i}, \ldots, \hat{\alpha}_{j}, \ldots,\left|\alpha_{i}-\alpha_{j}\right|\right) .
\end{aligned}
$$

These spaces $\mathcal{M}\left(\alpha_{i, j}^{ \pm}\right)$are themselves endowed with circle bundles and their Chern classes which we denote by $c_{i}^{ \pm}$. Considering for simplicity $i=m-1$ and $j=m$ (given a permutation $\sigma \in S y m_{m}$ there is an isomorphism between $\mathcal{M}(\alpha)$ and $\mathcal{M}\left(\alpha^{\sigma}\right)$ given by reordering the steps), in Proposition 5.1 we study the behavior of the different classes $c_{i}$ when restricted to $\left[D_{m-1, m}^{+}(\alpha)\right]$ and $\left[D_{m-1, m}^{-}(\alpha)\right]$, and we compare it with the behavior of $c_{i}^{ \pm}$. We then obtain the following recursion relation (cf. Section 5).

Theorem 1.1. Suppose $\alpha_{m} \neq \alpha_{m-1}$ and let

$$
\alpha^{+}:=\left(\alpha_{1}, \ldots, \alpha_{m-2}, \alpha_{m-1}+\alpha_{m}\right) \quad \text { and } \quad \alpha^{-}:=\left(\alpha_{1}, \ldots, \alpha_{m-2},\left|\alpha_{m-1}-\alpha_{m}\right|\right) .
$$

Then, for $k_{1}, \ldots, k_{m} \in \mathbb{Z}_{\geq 0}$ such that $k_{1}+\cdots+k_{m}=m-3$ and $k_{m} \geq 1$,

$$
\begin{aligned}
& \int_{\mathcal{M}(\alpha)} c_{1}^{k_{1}} \cdots c_{m}^{k_{m}}=\int_{\mathcal{M}\left(\alpha^{+}\right)}\left(c_{1}^{+}\right)^{k_{1}} \cdots\left(c_{m-2}^{+}\right)^{k_{m-2}}\left(c_{m-1}^{+}\right)^{k_{m-1}+k_{m}-1} \\
& +(-1)^{k_{m}-1}\left(\operatorname{sgn}\left(\alpha_{m-1}-\alpha_{m}\right)\right)^{k_{m-1}+k_{m}} \int_{\mathcal{M}\left(\alpha^{-}\right)}\left(c_{1}^{-}\right)^{k_{1}} \cdots\left(c_{m-2}^{-}\right)^{k_{m-2}}\left(c_{m-1}^{-}\right)^{k_{m-1}+k_{m}-1} .
\end{aligned}
$$

This recursion relation is an analog of the recursion relation appearing in the work of Witten and Kontsevich on moduli spaces of punctured curves [15, 24, 26] and on the work of Weitsman on moduli spaces of flat connections on twomanifolds of genus $g$ with $m$ marked points [23]. This is not surprising since for small values of $\alpha$ the polygon spaces $\mathcal{M}(\alpha)$ can be identified with moduli spaces of flat $S U(2)$-connections on the $m$-punctured sphere (cf. Section 9). The proof of Theorem 1.1 takes profit from this identification as it follows Weitsman's proof 23. of the corresponding recursion relation for moduli spaces of flat connections on surfaces of genus $g$, adapting it to the context of polygon spaces and making the necessary changes for the genus $g=0$ situation. 
Based on this recursion relation we obtain the following explicit expression for the computation of the intersection numbers (cf. Section 6 for details). Note that this result allows us to compute all intersection numbers since, given a permutation $\sigma \in$ $\mathrm{Sym}_{m}$, the natural isomorphism between $\mathcal{M}(\alpha)$ and $\mathcal{M}\left(\alpha^{\sigma}\right)$ induces an isomorphism on cohomology taking $c_{j}$ to $c_{\sigma^{-1}(j)}$ (cf. Remark 3.1).

Theorem 1.2. Let $\alpha=\left(\alpha_{1}, \ldots, \alpha_{m}\right)$ be generic. Suppose $k_{m-l}, \ldots, k_{m} \in \mathbb{Z}_{+}$, $k_{1}=\cdots=k_{m-l-1}=0$ and $k_{m-l}+\cdots+k_{m}=m-3$. Let $c_{i}:=c_{1}\left(V_{i}(\alpha)\right)$ be the first Chern classes of the circle bundles $V_{i}(\alpha) \rightarrow \mathcal{M}(\alpha)$. Then

$$
\int_{\mathcal{M}(\alpha)} c_{m-l}^{k_{m-l}} \cdots c_{m}^{k_{m}}=\sum_{J \in \mathcal{T}(\alpha)}(-1)^{\left(\sum_{i \in I \backslash J} k_{i}\right)+m-|J|} .
$$

Here $\mathcal{T}(\alpha)$ is a special family of sets $J \subset I:=\{3, \ldots, m\}$ that we will call triangular for which

$$
l_{J}:=\sum_{i=3}^{m}(-1)^{\chi_{I \backslash J}(i)} \alpha_{i}=\sum_{i \in J} \alpha_{i}-\sum_{i \in I \backslash J} \alpha_{i}>0,
$$

and for which the following triangle inequalities are satisfied:

$$
\begin{aligned}
& \alpha_{1} \leq \alpha_{2}+l_{J}, \\
& \alpha_{2} \leq \alpha_{1}+l_{J}, \\
& l_{J} \leq \alpha_{1}+\alpha_{2} .
\end{aligned}
$$

Alternatively, using the concept of short and long subsets introduced by Walker in [22, where $S \subset\{1, \ldots, m\}$ is called short if

$$
\sum_{i \in S} \alpha_{i}<\sum_{i \notin S} \alpha_{i}
$$

and long otherwise, $\mathcal{T}(\alpha)$ is the family of sets $J \subset I$ such that $J$ is short and both $J \cup\{1\}$ and $J \cup\{2\}$ are long.

After we prove this relation we work out several examples in Section 7 and compare the results obtained through our formulas with the ones obtained by Hausmann and Knutson in [8]. Then, in Section 8, we study the equilateral case $\mathcal{M}_{m}$. Requiring $\alpha$ to be generic in this case means exactly that $m$ is odd. The intersection numbers for these particular spaces have been determined by Kamiyama and Tezuka in [17] and are here presented in Theorem 8.1. We use Theorem 1.2 to compute these numbers, and we show the equivalence between the two results through several combinatorial computations. For completion, we consider the quotient of $\mathcal{M}_{m}$ by the action of the symmetric group. The cohomology ring of this space was computed by Brion [1, Klyachko [13] and Hausmann and Knutson 8]. It can be identified with the invariant part of $H^{*}\left(\mathcal{M}_{m}, ; \mathbb{Q}\right)$ by the symmetric group action and is generated by $\sigma_{1}$, the first invariant symmetric polynomial in the classes $c_{i}$, and, for example, by $c_{m}^{2}$, a generator of degree 4 (or any other $c_{i}^{2}$ since they are all equal to the Pontrjagin class of the principal $S O(3)$-bundle $A_{m} \rightarrow \mathcal{M}_{m}$, where $\left.A_{m}:=\left\{\left(\vec{v}_{1}, \ldots, \vec{v}_{m}\right) \in\left(\mathbb{R}^{3}\right)^{m}\left|\sum_{i=1}^{m} \vec{v}_{i}=0,\right| \vec{v}_{1}|=\cdots=| \vec{v}_{m} \mid\right\}\right)$. We compute the intersection numbers $\sigma_{1}^{k} \cdot c_{m}^{m-3-k}$ for an even $k$ and obtain the following result. 
Proposition 1.3. For an even integer $k$

$$
\begin{aligned}
& \int_{\mathcal{M}_{m} / S_{y m}} \sigma_{1}^{k} \cdot c_{m}^{m-3-k} \\
& =(-1)^{\frac{m-3}{2}}\left(\begin{array}{c}
m-2 \\
\frac{m-1}{2}
\end{array}\right) \sum_{j=0}^{\frac{k}{2}}(-1)^{j} \frac{\left(\begin{array}{c}
\frac{m-3}{2} \\
j
\end{array}\right)}{\left(\begin{array}{c}
m-2 \\
2 j
\end{array}\right)} \sum_{\begin{array}{l}
k_{1}+\cdots+k_{m}=k \text { s.t. } \\
2 j \text { of the } k_{i} \text { 's are odd }
\end{array}}\left(\begin{array}{c}
k \\
k_{1}, \cdots, k_{m}
\end{array}\right) .
\end{aligned}
$$

In Section 9 we explore the identification between polygon spaces and moduli spaces of flat $S U(2)$-connections on the $m$-punctured sphere and we relate the generating function for intersection pairings obtained by Yoshida 27 in the context of the moduli space of flat connections with our explicit formula from Theorem 1.2 .

Finally, in Section 10 we compare Theorem 1.2 with a formula for intersection pairings on polygon spaces previously obtained by Takakura 20 and Konno 14 by different methods. In particular, Takakura in 20 uses the "quantization commutes with reduction" theorem of Guillemin and Sternberg [5] to obtain an explicit formula for intersection pairings on polygon spaces, and Konno in 14 computes these pairings from an algebro-geometric point of view generalizing the methods of Kamiyama and Tezuka [17. The two expressions are equivalent (see [14) although Konno uses a different basis,

$$
v_{i}:=\frac{c_{i}+c_{m}}{2}, i=1, \ldots, m
$$

to write his formula.

We will show that their formula (here presented in Theorem 10.1) is equivalent to ours. Note, however, that our formula uses a smaller family of sets (the triangular sets $\mathcal{T}(\alpha)$ ) which is contained in the family of short sets $\mathcal{S}(\alpha)$ used in their result. Thus Theorem 1.2 greatly reduces the computations compared to KonnoTakakura's formula. For instance, if we consider $\alpha=(1, \ldots, 1, m-2)$ as in Example 7.2. the sum in our explicit formula has one term while that of Konno-Takakura has $2^{m-1}$ terms in all cases. Also, in Example 7.3 where $\alpha=(\varepsilon, \ldots, \varepsilon, 1,1,1)$, when $\mathcal{T}=\varnothing$ our sum has 0 terms while Konno-Takakura's needs $2^{m-1}$ terms to find 0.

Note also that Konno's method (like the one used in [17) relies on the explicit relations among the generators of the cohomology ring. Instead, our approach and Takakura's do not use these relations and contain enough information to recover the structure of this ring.

\section{Setting AND A BRIEF SURVEy on POLYGon SPACES}

Let $\alpha=\left(\alpha_{1}, \ldots, \alpha_{m}\right) \in \mathbb{R}_{+}^{m}$ and, for each $1 \leq i \leq m$, let $S_{\alpha_{i}}^{2}$ be the sphere of radius $\alpha_{i}$ in $\mathbb{R}^{3}$. The product $\prod_{i=1}^{m} S_{\alpha_{i}}^{2}$ can be thought of as the space of all paths starting at the origin with $m$ consecutive steps $\vec{v}_{i}$ whose lengths are $\left|\vec{v}_{i}\right|=\alpha_{i}$. We are interested in closed polygonal paths, i.e.,

$$
\left\{\vec{v}=\left(\vec{v}_{1}, \ldots, \vec{v}_{m}\right) \in \prod_{i=1}^{m} S_{\alpha_{i}}^{2} \mid \sum_{i=1}^{m} \vec{v}_{i}=0\right\} .
$$

The polygon space $\mathcal{M}(\alpha)$ is the space of all configurations of closed polygonal paths

in $\mathbb{R}^{3}$ starting and ending at the origin with $m$ edges of lengths $\alpha_{1}, \ldots, \alpha_{m}$, modulo 
rotation. More precisely,

$$
\mathcal{M}(\alpha):=\left\{\vec{v}=\left(\vec{v}_{1}, \ldots, \vec{v}_{m}\right) \in \prod_{i=1}^{m} S_{\alpha_{i}}^{2} \mid \sum_{i=1}^{m} \vec{v}_{i}=0\right\} / S O(3) .
$$

We want to rule out degenerate polygons, that is, polygons which are contained in a straight line. This degeneracy condition is equivalent to finding $\varepsilon_{i}= \pm 1$ for $1 \leq i \leq m$, such that

$$
\sum_{i=1}^{m} \varepsilon_{i} \alpha_{i}=0
$$

Hence, we say that $\alpha$ is generic if equation (2.1) has no solution with $\varepsilon_{i}= \pm 1$. If $\alpha$ is generic, the quotient space $\mathcal{M}(\alpha)$ is a symplectic manifold of dimension $2(m-3)$. Indeed, considering the product symplectic structure $\omega=\alpha_{1} \omega_{S^{2}}+\cdots+\alpha_{m} \omega_{S^{2}}$ on $\prod_{i=1}^{m} S_{\alpha_{i}}^{2} \subset\left(\mathbb{R}^{3}\right)^{m}$, where $\omega_{S^{2}}$ is the standard symplectic structure on $S^{2}$, and the diagonal Hamiltonian $S O(3)$-action with moment map

$$
\begin{aligned}
\mu: \quad \prod_{i=1}^{m} S_{\alpha_{i}}^{2} & \longrightarrow \mathfrak{s o}(3)^{*} \cong\left(\mathbb{R}^{3}\right)^{*}, \\
\vec{v}=\left(\vec{v}_{1}, \ldots, \vec{v}_{m}\right) & \longmapsto \mu(\vec{v})=\sum_{i=1}^{m} \vec{v}_{i} \quad \text { ("endpoint") },
\end{aligned}
$$

we can, for a generic $\alpha$, see the polygon space $\mathcal{M}(\alpha)$ as a symplectic quotient of the path space. When $\alpha$ is generic the $S O(3)$-action is free and 0 is a regular value of $\mu$, and so $\mathcal{M}(\alpha)$ is itself a symplectic manifold. More precisely,

$$
\mathcal{M}(\alpha)=\mu^{-1}(0) / S O(3)=\left(\prod_{i=1}^{m} S_{\alpha_{i}}^{2}\right) / /{ }_{0} S O(3) \cong \prod_{i=1}^{m-1} S_{\alpha_{i}}^{2} / \int_{\alpha_{m}} S O(3),
$$

where this last space is the set of paths of $m-1$ steps of lengths $\alpha_{1}, \ldots, \alpha_{m-1}$ whose endpoint is at a distance $\alpha_{m}$ from the origin modulo $S O(3)$ (see [13, 16, 8], for additional details). Hereafter, we will assume that $\alpha$ is generic.

Remark 2.1. For $\alpha$ and $\alpha^{\prime}$ generic and sufficiently close, $\mathcal{M}(\alpha)$ and $\mathcal{M}\left(\alpha^{\prime}\right)$ are diffeomorphic ([8], Proposition 2.2). Indeed, if they are close enough such that $\alpha(t):=t \alpha^{\prime}+(1-t) \alpha$ is always generic for $t \in[0,1]$, the map

$$
\begin{aligned}
\beta: & {[0,1] \times \prod_{i=1}^{m} S^{2} \rightarrow[0,1] \times \mathbb{R}^{3}, } \\
\left(t, z_{1}, \ldots, z_{m}\right) & \mapsto\left(t, \mu\left(\alpha_{1}(t) z_{1}, \ldots, \alpha_{m}(t) z_{m}\right)\right)
\end{aligned}
$$

has no critical values in $[0,1] \times\{0\}$, and so its gradient flow gives an $S O(3)$ equivariant diffeomorphism between $\beta^{-1}(0,0)$ and $\beta^{-1}(1,0)$. Hence, the quotient spaces $\mathcal{M}(\alpha)=\beta^{-1}(0,0) / S O(3)$ and $\mathcal{M}\left(\alpha^{\prime}\right)=\beta^{-1}(1,0) / S O(3)$ are also diffeomorphic. 
The restriction of the diagonal $S O(3)$-action on the path space to an action of $S^{1} \cong S O(2)$ is also Hamiltonian with moment map

$$
\begin{aligned}
\bar{\mu}: \quad \prod_{i=1}^{m} S_{\alpha_{i}}^{2} \quad \longrightarrow \mathfrak{s o}(2)^{*} \cong(\mathbb{R})^{*}, \\
\vec{v}=\left(\vec{v}_{1}, \ldots, \vec{v}_{m}\right) \longmapsto \bar{\mu}(\vec{v})=\zeta\left(\sum_{i=1}^{m} \vec{v}_{i}\right) \quad \text { ("height of endpoint") }
\end{aligned}
$$

where $\zeta$ is the projection $\zeta(x, y, z)=z$.

Using the maps $\zeta$ and $\bar{\mu}$ we will define some other spaces that will be relevant to us. The first is the abelian polygon space,

$$
\mathcal{A M}(\alpha):=\left\{\vec{v}=\left(\vec{v}_{1}, \ldots, \vec{v}_{m-1}\right) \in \prod_{i=1}^{m-1} S_{\alpha_{i}}^{2} \mid \zeta\left(\sum_{i=1}^{m-1} \vec{v}_{i}\right)=\alpha_{m}\right\} / S^{1},
$$

the space of piecewise linear $(m-1)$-chains with edge lengths $\alpha_{1}, \ldots, \alpha_{m-1}$ which end on the plane $z=\alpha_{m}$, modulo rotations around the $z$-axis. It is a symplectic quotient of the path space $\prod_{i=1}^{m-1} S_{\alpha_{i}}^{2}$ by the maximal torus $S^{1}$ of $S O(3)$,

$$
\mathcal{A M}(\alpha)=\zeta^{-1}\left(\alpha_{m}\right) / S^{1},
$$

and so it is a symplectic manifold of dimension $2(m-2)$ containing $\mathcal{M}(\alpha)$ as a symplectic submanifold of codimension 2 .

Remark 2.2. Note that we can always rotate any element $\vec{v} \in \mathcal{M}(\alpha)$ in such a way that the sum $\sum_{i=1}^{m-1} \vec{v}_{i}$ ends not only on the plane $z=\alpha_{m}$ but also on the $z$-axis, so that $\vec{v}_{m}$ points downwards.

The upper path space $\mathcal{U P}(\alpha)$ is defined as the space

$$
\mathcal{U P}(\alpha):=\left\{\vec{v}=\left(\vec{v}_{1}, \ldots, \vec{v}_{m-1}\right) \in \prod_{i=1}^{m-1} S_{\alpha_{i}}^{2} \mid \zeta\left(\sum_{i=1}^{m-1} \vec{v}_{i}\right) \geq \alpha_{m}\right\} / \sim,
$$

where $\vec{v} \sim \vec{v}^{\prime}$ if and only if $\vec{v}=\vec{v}^{\prime}$ or $\zeta\left(\sum_{i=1}^{m-1} \vec{v}_{i}\right)=\alpha_{m}$ and $[\vec{v}]=\left[\vec{v}^{\prime}\right]$ in $\mathcal{A M}(\alpha)$. It is the symplectic cut (in the sense of [18]) of the path space $\prod_{i=1}^{m-1} S_{\alpha_{i}}^{2}$ at the level $\alpha_{m}$ of the moment map $\bar{\mu}$. Hence, it is a symplectic manifold of dimension $2(m-1)$ which contains $\mathcal{A M}(\alpha)$ as a submanifold of codimension 2.

\section{Circle Bundles over Polygon spaces}

We construct circle bundles over $\mathcal{M}(\alpha)$ as follows. We define, for each $1 \leq j \leq m$, the space

$$
V_{j}(\alpha):=\left\{\vec{v}=\left(\vec{v}_{1}, \ldots, \vec{v}_{m}\right) \in \prod_{i=1}^{m} S_{\alpha_{i}}^{2} \mid \sum_{i=1}^{m} \vec{v}_{i}=0 \text { and } \vec{v}_{j}=\left(0,0, \alpha_{j}\right)\right\}
$$

which is a smooth manifold of dimension $2 m-5$. The circle $S^{1}$ acts on $V_{j}(\alpha)$ by rotation around the $z$-axis. As $\alpha$ is generic, this action is free, and it is easy to check that $V_{j}(\alpha) / S^{1}=\mathcal{M}(\alpha)$. Hence, $V_{j}(\alpha) \rightarrow \mathcal{M}(\alpha)$ is a principal circle bundle determined by its Chern class

$$
c_{j}:=c_{1}\left(V_{j}(\alpha)\right) \in H^{2}(\mathcal{M}(\alpha) ; \mathbb{Z}) .
$$


Let us also consider the level set $B(\alpha):=\zeta^{-1}\left(\alpha_{m}\right) \subset \prod_{i=1}^{m-1} S_{\alpha_{i}}^{2}$, i.e.

$$
B(\alpha):=\left\{\vec{v}=\left(\vec{v}_{1}, \ldots, \vec{v}_{m-1}\right) \in \prod_{i=1}^{m-1} S_{\alpha_{i}}^{2} \mid \zeta\left(\sum_{i=1}^{m-1} \vec{v}_{i}\right)=\alpha_{m}\right\} .
$$

Since $\alpha$ is generic, $S^{1}$ acts freely on this space making it a principal bundle over $\mathcal{A M}(\alpha)$. Moreover, we have the following commutative diagram:

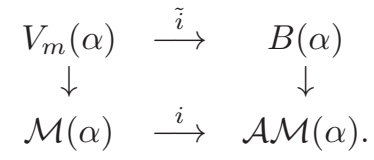

Note that the inclusion $\tilde{i}: V_{m}(\alpha) \hookrightarrow B(\alpha)$ is anti-equivariant (i.e. $\tilde{i}(\lambda \cdot \vec{v})=\lambda^{-1} \cdot \tilde{i}(\vec{v})$ ) since, in the identification of $\mathcal{M}(\alpha)$ as a submanifold of $\mathcal{A} \mathcal{M}(\alpha)$, the vector $\vec{v}_{m}$ must face downward (see Remark 2.2). Hence, $c_{m}=-i^{*}\left(c_{1}(B(\alpha))\right)$.

Let $\mathcal{N}$ be a tubular neighborhood of $\mathcal{A M}(\alpha)$ inside $\mathcal{U P}(\alpha)$. The retraction $\mathcal{N} \rightarrow \mathcal{A M}(\alpha)$ is the disc bundle associated to the circle bundle $B(\alpha) \rightarrow \mathcal{A M}(\alpha)$, and so $c_{1}(B(\alpha))$ is the Euler class of the normal bundle of $\mathcal{A M}(\alpha)$ inside $\mathcal{U P}(\alpha)$. On the other hand, since $\mathcal{A M}(\alpha)$ is the reduced space (2.2),

$$
\zeta^{-1}\left(\alpha_{m}\right) / S^{1}
$$

we have, by the Duistermaat-Heckmann theorem [2, 3], that

$$
c_{1}(B(\alpha))=-\frac{\partial}{\partial \alpha_{m}}[\omega]
$$

in $H^{2}(\mathcal{A M}(\alpha) ; \mathbb{R})$, and so

$$
c_{m}=\frac{\partial}{\partial \alpha_{m}}[\omega]
$$

in $H^{2}(\mathcal{M}(\alpha) ; \mathbb{R})$. By symmetry, since any edge can be the last, we have

$$
c_{j}=\frac{\partial}{\partial \alpha_{j}}[\omega]
$$

It is shown in 8 ] that these classes $c_{j}$ generate $H^{*}\left(\mathcal{M}(\alpha) ; \mathbb{Z}\left[\frac{1}{2}\right]\right)$ but do not generate $H^{*}(\mathcal{M}(\alpha) ; \mathbb{Z})$.

Remark 3.1. Given a permutation $\sigma \in \mathrm{Sym}_{m}$ there is an isomorphism between $\mathcal{M}(\alpha)$ and $\mathcal{M}\left(\alpha^{\sigma}\right)$ given by reordering the steps (note that a polygon is simply a list of $m$ vectors whose sum is zero modulo rotation). From the above geometric construction of the bundles $V_{j}(\alpha)$ we see that the induced isomorphism on cohomology $H^{2}\left(\mathcal{M}\left(\alpha^{\sigma}\right)\right) \rightarrow H^{2}(\mathcal{M}(\alpha))$ yields $c_{j} \rightarrow c_{\sigma^{-1}(j)}$.

\section{Dual homology Classes}

The content of the next two sections takes profit from the identification, for small values of $\alpha$, between polygon spaces $\mathcal{M}(\alpha)$ and moduli spaces of flat $S U(2)$ connections on the $m$-punctured sphere (cf. Section 9). Indeed, we follow the proof of Weitsman's recursion relation 23] for moduli spaces of flat connections on surfaces of genus $g$, adapting it to the context of polygon spaces and making the necessary changes for the genus $g=0$ situation.

We are interested in determining homology cycles representing the Poincaré duals of the first Chern classes $c_{j}:=c_{1}\left(V_{j}(\alpha)\right) \in H^{2}(\mathcal{M}(\alpha) ; \mathbb{Z})$. For this purpose, let $i$ 
and $j$ be positive integers such that $1 \leq i, j \leq m$ and $i \neq j$. Let us denote by $D_{i, j}(\alpha)$ the submanifold of $\mathcal{M}(\alpha)$ formed by those polygons $\vec{v} \in \mathcal{M}(\alpha)$ for which the step $\vec{v}_{i}$ is parallel to $\vec{v}_{j}$. By rotating $\vec{v}$ around a suitable axis, if necessary, we can assume that $\vec{v}_{i}$ and $\vec{v}_{j}$ are parallel to the $z$ axis. Hence

$$
D_{i, j}(\alpha):=\left\{\vec{v}=\left(\vec{v}_{1}, \ldots, \vec{v}_{m}\right) \in \mathcal{M}(\alpha) \mid \vec{v}_{i} \text { and } \vec{v}_{j} \text { are linearly dependent }\right\}
$$

is a codimension two submanifold of $\mathcal{M}(\alpha)$. We will show that the bundle $V_{j}(\alpha)$ has a section on the complement of $D_{i, j}(\alpha)$, and so $c_{j}$ will be dual to an element of $i_{*}\left(H_{2(n-4)}\left(D_{i, j}(\alpha)\right)\right)$, where $i: D_{i, j}(\alpha) \hookrightarrow \mathcal{M}(\alpha)$ is the inclusion map. Note that $D_{i, j}(\alpha)=D_{j, i}(\alpha)$, so $D_{i, j}(\alpha)$ will not be connected. Indeed, both $V_{i}(\alpha)$ and $V_{j}(\alpha)$ are trivial on the complement of $D_{i, j}(\alpha)$, but they are not powers of the same circle bundle.

Proposition 4.1. The circle bundle $\left.V_{j}(\alpha)\right|_{\mathcal{M}(\alpha) \backslash D_{i, j}(\alpha)} \stackrel{\pi_{j}}{\longrightarrow} \mathcal{M}(\alpha) \backslash D_{i, j}(\alpha)$ has a section.

Proof. A section $s: \mathcal{M}(\alpha) \backslash D_{i, j}(\alpha) \rightarrow V_{j}(\alpha)$ can be given by choosing, for each $\vec{v} \in \mathcal{M}(\alpha)$, a representative $s(\vec{v}) \in V_{j}(\alpha)$ such that $\pi_{j}(s(\vec{v}))=\vec{v}$. To do this we assign to each $\vec{v}$ the unique element $p \in \pi_{j}^{-1}(\vec{v}) \subset V_{j}(\alpha)$ for which the $i$-th step $\vec{v}_{i}$ projects onto the $x O y$ plane along the positive $x$-axis. Such a representative will always exist in $\pi_{j}^{-1}(\vec{v})$ as long as $\vec{v}_{i}$ is not parallel to $\vec{v}_{j}$, that is, as long as $\vec{v} \notin D_{i, j}(\alpha)$.

Since $D_{i, j}(\alpha)$ consists of classes of polygons for which the step $\vec{v}_{i}$ is parallel to $\vec{v}_{j}$, it clearly has two different connected components,

$D_{i, j}^{+}(\alpha)=\left\{\vec{v} \in D_{i, j}(\alpha) \mid\left\langle\vec{v}_{i}, \vec{v}_{j}\right\rangle>0\right\} \quad$ and $\quad D_{i, j}^{-}(\alpha)=\left\{\vec{v} \in D_{i, j}(\alpha) \mid\left\langle\vec{v}_{i}, \vec{v}_{j}\right\rangle<0\right\}$.

Both spaces $D_{i, j}^{ \pm}(\alpha)$ are symplectomorphic to polygon spaces obtained by permuting the edges so that the steps $\vec{v}_{i}$ and $\vec{v}_{j}$ become the last two consecutive edges. In other words,

Proposition 4.2. For $\alpha_{i} \neq \alpha_{j}$ there exist symplectomorphisms

$$
\begin{aligned}
& s_{ \pm}: D_{i, j}^{ \pm}(\alpha) \\
& {\left[\left(\vec{v}_{1}, \ldots, \vec{v}_{m}\right)\right] } \mapsto \mathcal{M}\left(\alpha_{i, j}^{ \pm}\right), \\
& {\left[\left(\vec{v}_{1}, \ldots, \hat{\vec{v}}_{i}, \ldots, \hat{\vec{v}}_{j}, \ldots, \vec{v}_{i} \pm \vec{v}_{j}\right)\right], }
\end{aligned}
$$

where

$$
\begin{aligned}
\alpha_{i, j}^{+} & =\left(\alpha_{1}, \ldots, \hat{\alpha_{i}}, \ldots, \hat{\alpha_{j}}, \ldots, \alpha_{i}+\alpha_{j}\right), \\
\alpha_{i, j}^{-} & =\left(\alpha_{1}, \ldots, \hat{\alpha_{i}}, \ldots, \hat{\alpha_{j}}, \ldots,\left|\alpha_{i}-\alpha_{j}\right|\right) .
\end{aligned}
$$

Note that both $\alpha_{i, j}^{+}$and $\alpha_{i, j}^{-}$are generic provided that $\alpha$ is generic. We conclude that the manifolds $D_{i, j}^{ \pm}(\alpha)$ are connected symplectic manifolds, and so we can orient them using the symplectic form by requiring that $\int_{D_{i, j}^{ \pm}(\alpha)} \omega^{n-4}>0$. We obtain in this way two generators of $H_{2(n-4)}\left(D_{i, j}^{ \pm}(\alpha)\right):\left[D_{i, j}^{+}(\alpha)\right]$ and $\left[D_{i, j}^{-}(\alpha)\right]$. Therefore, to determine the Poincaré dual of $c_{j}$ we just have to determine two constants $a_{i, j}$ and $b_{i, j}$ as in the following proposition.

Proposition 4.3. Let $i: D_{i, j}(\alpha) \hookrightarrow \mathcal{M}(\alpha)$ be the inclusion map. If $\alpha_{i} \neq \alpha_{j}$, then the Poincaré dual of $c_{j}$ is in $i_{*} H_{2(n-4)}\left(D_{i, j}(\alpha)\right)$ and can be written as

$$
a_{i, j}\left[D_{i, j}^{+}(\alpha)\right]+b_{i, j}\left[D_{i, j}^{-}(\alpha)\right] .
$$


We will now see that the constants $a_{i, j}$ are always equal to 1 , while the constants $b_{i, j}$ depend on the values of $\alpha_{i}$ and $\alpha_{j}$.

Proposition 4.4. The constants $a_{i, j}$ and $b_{i, j}$ are equal to

$$
a_{i, j}=1 \quad \text { and } \quad b_{i, j}=\operatorname{sgn}\left(\alpha_{i}-\alpha_{j}\right) .
$$

Proof. For simplicity, we consider $i=m-1$ and $j=m$ (we can always reduce to this case due to Remark 3.1). Take a fixed polygon $\underline{\vec{v}}$ in $\mathbb{R}^{3}$ such that $\left|\underline{\vec{v}}_{i}\right|=\alpha_{i}$ and consider the space

$$
\begin{gathered}
W:=\left\{\vec{v}=\left(\vec{v}_{1}, \ldots, \vec{v}_{m}\right) \in\left(\mathbb{R}^{3}\right)^{m}\left|\quad \sum_{k=1}^{m} \vec{v}_{k}=0, \quad\right| \vec{v}_{k} \mid=\alpha_{k}, \quad k=1, \ldots, m,\right. \\
\text { and } \left.\quad \vec{v}_{i}=\underline{\vec{v}}_{i}, \quad i=1, \ldots, m-3\right\} / S O(3) .
\end{gathered}
$$

This corresponds to fixing the first $m-3$ edges of the polygons, allowing one to move only the last three. The manifold $W$ is symplectomorphic to the sphere $\mathcal{M}\left(l, \alpha_{m-2}, \alpha_{m-1}, \alpha_{m}\right)$, where $l:=\left|\sum_{k=1}^{m-3} \underline{\vec{v}}_{k}\right|$. To find the constants $a_{i, j}$ and $b_{i, j}$ we just have to compare $\left.c_{m}\right|_{W}$ with $W \cap D_{m-1, m}^{ \pm}(\alpha)$.

We compute $\left.c_{m}\right|_{W}$ using the variation of the symplectic form given in (3.1). Hence, if $i: W \hookrightarrow \mathcal{M}(\alpha)$ is the inclusion map, then

$$
\left.c_{m}\right|_{W}=i^{*} c_{m}=i^{*} \frac{\partial}{\partial \alpha_{m}}[\omega]=\frac{\partial}{\partial \alpha_{m}}\left[i^{*} \omega\right] .
$$

On the other hand, $W$ is a toric manifold (equivariantly symplectomorphic to a sphere equipped with a circle action) with a moment map

$$
\mu: W \cong \mathcal{M}\left(l, \alpha_{m-2}, \alpha_{m-1}, \alpha_{m}\right) \rightarrow \mathbb{R}^{*}
$$

obtained by restricting to $W$ the action on $\mathcal{M}(\alpha)$ given by bending the polygons along the last diagonal. The moment map image $\mu(W)$ is the interval

$$
\Delta=\left[\max \left\{\left|l-\alpha_{m-2}\right|,\left|\alpha_{m-1}-\alpha_{m}\right|\right\}, \min \left\{l+\alpha_{m-2}, \alpha_{m-1}+\alpha_{m}\right\}\right]
$$

(see Section 6 of 7 for details).

Now let $M^{2 n}$ be an arbitrary toric manifold with moment map $\mu$, equipped with a family of symplectic forms $\Omega_{t}$. The corresponding family of moment polytopes $\Delta_{t} \subset \mathfrak{t}^{*}$ is determined by their facets

$$
F_{k}=\left\{x \in \mathbb{R}^{n} \mid\left\langle x, u_{k}\right\rangle=\lambda_{k}(t)\right\}, \quad k=1, \ldots, N,
$$

where $N$ is the number of facets of $\Delta_{t}$. Suppose that the polytopes $\Delta_{t}$ stay combinatorially the same as $t$ changes but the value $\lambda_{i}(t)$ for some $i \in\{1, \ldots, N\}$ depends linearly on $t$ and, as $t$ increases, the facet $F_{i}$ moves outward while the others stay fixed. Then we know that $\frac{d}{d t} \Omega_{t}$ is the Poincaré dual of the homology class $\left[\mu^{-1}\left(F_{i}\right)\right]$, where the orientation of $\mu^{-1}\left(F_{i}\right)$ is given by requiring $\int_{\mu^{-1}\left(F_{i}\right)} \Omega_{t}^{n-1}>0$ (cf. Section 2.2 of [3] for details).

Applying this general fact about toric manifolds to $W$ and its moment polytope $\Delta$, we see that as $\alpha_{m}$ changes, the cohomology class of the symplectic form of $W$, $\left[\omega_{\alpha_{m}}\right]$, changes by the Poincaré dual of the homology class

$$
\begin{aligned}
& {\left[\mu^{-1}\left(\alpha_{m-1}+\alpha_{m}\right)\right]+\operatorname{sgn}\left(\alpha_{m-1}-\alpha_{m}\right)\left[\mu^{-1}\left(\left|\alpha_{m-1}-\alpha_{m}\right|\right)\right]} \\
& =\left[D_{m-1, m}^{+}(\alpha) \cap W\right]+\operatorname{sgn}\left(\alpha_{m-1}-\alpha_{m}\right)\left[D_{m-1, m}^{-}(\alpha) \cap W\right],
\end{aligned}
$$

and the result follows. 


\section{A RECURSion FORMUla}

In order to prove Theorem 1.1 we still need to study the behavior of the different Chern classes $c_{i}$ when restricted to $\left[D_{m-1, m}^{+}(\alpha)\right]$ and $\left[D_{m-1, m}^{-}(\alpha)\right]$.

Proposition 5.1. Suppose $\alpha_{m} \neq \alpha_{m-1}$ and let $c_{m}^{+}$and $c_{m}^{-}$be the cohomology classes $c_{1}\left(V_{m}\left(\alpha^{+}\right)\right)$and $c_{1}\left(V_{m}\left(\alpha^{-}\right)\right)$, where

$$
\alpha^{+}:=\left(\alpha_{1}, \ldots, \alpha_{m-2}, \alpha_{m-1}+\alpha_{m}\right) \quad \text { and } \quad \alpha^{-}:=\left(\alpha_{1}, \ldots, \alpha_{m-2},\left|\alpha_{m-1}-\alpha_{m}\right|\right) .
$$

Then, considering the inclusion maps $i_{ \pm}: D_{m-1, m}^{ \pm}(\alpha) \hookrightarrow \mathcal{M}(\alpha)$ and the symplectomorphisms $s_{ \pm}: D_{i, j}^{ \pm}(\alpha) \longrightarrow \mathcal{M}\left(\alpha_{i, j}^{ \pm}\right)$from Proposition 4.2 , we have

$$
\begin{aligned}
& \left(i_{ \pm} \circ s_{ \pm}^{-1}\right)^{*} c_{i}=c_{i}^{ \pm} \quad \text { for } \quad 1 \leq i \leq m-2, \\
& \left(i_{+} \circ s_{+}^{-1}\right)^{*} c_{m-1}=c_{m-1}^{+}, \\
& \left(i_{-} \circ s_{-}^{-1}\right)^{*} c_{m-1}=\operatorname{sgn}\left(\alpha_{m-1}-\alpha_{m}\right) c_{m-1}^{-}, \\
& \left(i_{+} \circ s_{+}^{-1}\right)^{*} c_{m}=c_{m-1}^{+}, \\
& \left(i_{-} \circ s_{-}^{-1}\right)^{*} c_{m}=-\operatorname{sgn}\left(\alpha_{m-1}-\alpha_{m}\right) c_{m-1}^{-}
\end{aligned}
$$

Proof. Let us denote by $\omega^{+}$and $\omega^{-}$the symplectic forms on $\mathcal{M}\left(\alpha^{+}\right)$and $\mathcal{M}\left(\alpha^{-}\right)$ obtained by restricting to $D_{m-1, m}^{ \pm}(\alpha)$ the symplectic form $\omega$ of $\mathcal{M}(\alpha)$. Then, for $1 \leq i \leq m-2$, we have

$$
c_{i}^{ \pm}=\frac{\partial}{\partial \alpha_{i}^{ \pm}}\left[\omega^{ \pm}\right]=\frac{\partial}{\partial \alpha_{i}}\left[\left(i_{ \pm} \circ s_{ \pm}^{-1}\right)^{*} \omega\right]=\left(i_{ \pm} \circ s_{ \pm}^{-1}\right)^{*} \frac{\partial}{\partial \alpha_{i}}[\omega]=\left(i_{ \pm} \circ s_{ \pm}^{-1}\right)^{*} c_{i},
$$

since $\alpha_{i}^{ \pm}=\alpha_{i}$ for $1 \leq i \leq m-2$. Moreover,

$c_{m-1}^{+}=\frac{\partial}{\partial \alpha_{m-1}^{+}}\left[\omega^{+}\right]=\left(\frac{\partial}{\partial \alpha_{m-1}}\left[\omega^{+}\right]\right)\left(\frac{\partial \alpha_{m-1}}{\partial \alpha_{m-1}^{+}}\right)=\frac{\partial}{\partial \alpha_{m-1}}\left[\omega^{+}\right]=\left(i_{+} \circ s_{+}^{-1}\right)^{*} c_{m-1}$,

since $\alpha_{m-1}^{+}=\alpha_{m-1}+\alpha_{m}$. Similarly,

$$
\begin{aligned}
c_{m-1}^{-} & =\frac{\partial}{\partial \alpha_{m-1}^{-}}\left[\omega^{-}\right]=\left(\frac{\partial}{\partial \alpha_{m-1}}\left[\omega^{-}\right]\right)\left(\frac{\partial \alpha_{m-1}}{\partial \alpha_{m-1}^{-}}\right)=\frac{\partial}{\partial \alpha_{m-1}}\left[\omega^{-}\right] \cdot \operatorname{sgn}\left(\alpha_{m-1}-\alpha_{m}\right) \\
& =\operatorname{sgn}\left(\alpha_{m-1}-\alpha_{m}\right)\left(i_{-} \circ s_{-}^{-1}\right)^{*} c_{m-1},
\end{aligned}
$$

since $\alpha_{m-1}^{-}=\left|\alpha_{m-1}-\alpha_{m}\right|=\operatorname{sgn}\left(\alpha_{m-1}-\alpha_{m}\right)\left(\alpha_{m-1}-\alpha_{m}\right)$. On the other hand,

$$
c_{m-1}^{+}=\frac{\partial}{\partial \alpha_{m-1}^{+}}\left[\omega^{+}\right]=\left(\frac{\partial}{\partial \alpha_{m}}\left[\omega^{+}\right]\right)\left(\frac{\partial \alpha_{m}}{\partial \alpha_{m-1}^{+}}\right)=\frac{\partial}{\partial \alpha_{m}}\left[\omega^{+}\right]=\left(i_{+} \circ s_{+}^{-1}\right)^{*} c_{m}
$$

and

$$
\begin{aligned}
c_{m-1}^{-} & =\frac{\partial}{\partial \alpha_{m-1}^{-}}\left[\omega^{-}\right]=\left(\frac{\partial}{\partial \alpha_{m}}\left[\omega^{-}\right]\right)\left(\frac{\partial \alpha_{m}}{\partial \alpha_{m-1}^{-}}\right)=-\frac{\partial}{\partial \alpha_{m}}\left[\omega^{-}\right] \cdot \operatorname{sgn}\left(\alpha_{m-1}-\alpha_{m}\right) \\
& =-\operatorname{sgn}\left(\alpha_{m-1}-\alpha_{m}\right)\left(i_{-} \circ s_{-}^{-1}\right)^{*} c_{m} .
\end{aligned}
$$

Finally, using Propositions 4.4 and 5.1 we have our recursion formula (1.1). 
Proof of Theorem 1.1. By Proposition 4.4 the Poincaré dual of $c_{m}$ is

$$
i_{*}^{+}\left(\left[D_{m-1, m}^{+}\right]\right)+\operatorname{sgn}\left(\alpha_{m-1}-\alpha_{m}\right) i_{*}^{-}\left(\left[D_{m-1, m}^{-}\right]\right) .
$$

This means that the formula

$$
\int_{\mathcal{M}(\alpha)} a c_{m}=\int_{\mathcal{M}\left(\alpha^{+}\right)} i_{+}^{*}(a)+\operatorname{sgn}\left(\alpha_{m-1}-\alpha_{m}\right) \int_{\mathcal{M}\left(\alpha^{-}\right)} i_{-}^{*}(a)
$$

holds true for all $a \in H^{m-4}(\mathcal{M}(\alpha))$. Theorem 1.1 then follows from Proposition 5.1.

\section{An explicit FORMUla}

Using the recursion formula (1.1) we may obtain an explicit expression for the computation of intersection numbers. For that we first need to introduce the following definition.

Definition 6.1. Let $\alpha=\left(\alpha_{1}, \ldots, \alpha_{m}\right)$ be generic. A set $J \subset I:=\{3, \ldots, m\}$ is called triangular if

$$
l_{J}:=\sum_{i=3}^{m}(-1)^{\chi_{I \backslash J}(i)} \alpha_{i}>0
$$

(where, for a set $S, \chi_{S}: S \rightarrow\{0,1\}$ is the characteristic function of $S$ ) and satisfies the following triangle inequalities:

$$
\begin{aligned}
& \alpha_{1} \leq \alpha_{2}+l_{J}, \\
& \alpha_{2} \leq \alpha_{1}+l_{J}, \\
& l_{J} \leq \alpha_{1}+\alpha_{2}
\end{aligned}
$$

(or equivalently if $J$ is short and both $J \cup\{1\}$ and $J \cup\{2\}$ are long). Moreover, define $\mathcal{T}:=\mathcal{T}(\alpha):=\{J \subset I \mid J$ is triangular $\}$, the family of all triangular sets in $I$.

Finally we come to the proof of Theorem 1.2

Proof of Theorem 1.2, We will prove this formula by induction on $m$ starting with $m=4$.

For $m=4$, the recursion formula of Theorem 1.1 gives

$$
\int_{\mathcal{M}\left(\alpha_{1}, \ldots, \alpha_{4}\right)} c_{4}=\int_{\mathcal{M}\left(\alpha_{1}, \alpha_{2}, \alpha_{3}+\alpha_{4}\right)} 1+\operatorname{sgn}\left(\alpha_{3}-\alpha_{4}\right) \int_{\mathcal{M}\left(\alpha_{1}, \alpha_{2},\left|\alpha_{3}-\alpha_{4}\right|\right)} 1 .
$$

Hence,

$$
\int_{\mathcal{M}\left(\alpha_{1}, \ldots, \alpha_{4}\right)} c_{4}=\left\{\begin{array}{cll}
2 & \text { if } & \mathcal{T}=\{\{3\},\{3,4\}\} \\
0 & \text { if } & \mathcal{T}=\{\{4\},\{3,4\}\} \text { or } \varnothing \\
1 & \text { if } & \mathcal{T}=\{\{3\}\} \text { or }\{\{3,4\}\} \\
-1 & \text { if } & \mathcal{T}=\{\{4\}\}
\end{array}\right.
$$

and it is easy to verify that this agrees in all cases with the right-hand side of (1.2). Note that in (6.2) we have listed all possible cases since $\{3\}$ and $\{4\}$ cannot be simultaneous in $\mathcal{T}$. Indeed, this would imply $\left|\alpha_{1}-\alpha_{2}\right| \leq \alpha_{3}-\alpha_{4}$ and $\left|\alpha_{1}-\alpha_{2}\right| \leq$ $\alpha_{4}-\alpha_{3}$ (from the first two triangular inequalities in (6.1)), which is impossible since $\left|\alpha_{1}-\alpha_{2}\right|>0$. 
We will now assume that (1.2) holds for some $m$ and show that it is still valid for $m+1$. Using the recursion formula (1.1) once more we obtain

$$
\begin{gathered}
\int c_{m+1-l}^{k_{m+1}} \cdots c_{m+1}^{k_{m+1}}=\int\left(c_{m+1-l}^{+}\right)^{k_{m+1}-l} \cdots\left(c_{m-1}^{+}\right)^{k_{m-1}}\left(c_{m}^{+}\right)^{k_{m}+k_{m+1}-1} \\
\mathcal{M}\left(\alpha_{1}, \ldots, \alpha_{m}, \alpha_{m+1}\right) \\
\mathcal{M}\left(\alpha_{1}, \ldots, \alpha_{m-1}, \alpha_{m}+\alpha_{m+1}\right) \\
+(-1)^{k_{m+1}-1} \operatorname{sgn}\left(\alpha_{m}-\alpha_{m+1}\right)^{k_{m}+k_{m+1}} \int\left(c_{m+1-l}^{-}\right)^{k_{m+1-l}} \\
\mathcal{M}\left(\alpha_{1}, \ldots, \alpha_{m-1},\left|\alpha_{m}-\alpha_{m+1}\right|\right) \\
\cdots\left(c_{m-1}^{-}\right)^{k_{m-1}}\left(c_{m}^{-}\right)^{k_{m}+k_{m+1}-1}
\end{gathered}
$$

Writing $\mathcal{T}_{m+1}:=\mathcal{T}\left(\alpha_{1}, \ldots, \alpha_{m}, \alpha_{m+1}\right), \mathcal{T}_{m}^{+}:=\mathcal{T}\left(\alpha_{1}, \ldots, \alpha_{m-1}, \alpha_{m}+\alpha_{m+1}\right)$ and $\mathcal{T}_{m}^{-}=\mathcal{T}\left(\alpha_{1}, \ldots, \alpha_{m-1},\left|\alpha_{m}-\alpha_{m+1}\right|\right)$, then, if $\alpha_{m}-\alpha_{m+1}>0$,

$$
\begin{aligned}
\mathcal{T}_{m+1}= & \left\{\widetilde{J} \in \mathcal{T}_{m}^{+} \mid m \notin \widetilde{J}\right\} \cup\left\{\widetilde{J} \cup\{m+1\} \mid \widetilde{J} \in \mathcal{T}_{m}^{+} \text {and } m \in \widetilde{J}\right\} \\
& \cup\left\{\widetilde{J} \in \mathcal{T}_{m}^{-} \mid m \in \widetilde{J}\right\} \cup\left\{\widetilde{J} \cup\{m+1\} \mid \widetilde{J} \in \mathcal{T}_{m}^{-} \text {and } m \notin \widetilde{J}\right\},
\end{aligned}
$$

while, if $\alpha_{m}-\alpha_{m+1}<0$,

$$
\begin{aligned}
& \mathcal{T}_{m+1}=\left\{\widetilde{J} \in \mathcal{T}_{m}^{+} \mid m \notin \widetilde{J}\right\} \cup\left\{\widetilde{J} \cup\{m+1\} \mid \widetilde{J} \in \mathcal{T}_{m}^{+} \text {and } m \in \widetilde{J}\right\} \\
& \cup\left\{(\widetilde{J} \backslash\{m\}) \cup\{m+1\} \mid \widetilde{J} \in \mathcal{T}_{m}^{-} \text {and } m \in \widetilde{J}\right\} \cup\left\{\widetilde{J} \cup\{m\} \mid \widetilde{J} \in \mathcal{T}_{m}^{-} \text {and } m \notin \widetilde{J}\right\}
\end{aligned}
$$

Assuming (1.2) holds for $m$ and writing $\tilde{k}_{j}=k_{j}$ for $j \neq m$ and $\tilde{k}_{m}=k_{m}+k_{m+1}-1$, we have

$$
\begin{aligned}
\int & \left(c_{m+1-l}^{+}\right)^{k_{m+1}-l} \cdots\left(c_{m-1}^{+}\right)^{k_{m-1}}\left(c_{m}^{+}\right)^{k_{m}+k_{m+1}-1} \\
\mathcal{M}\left(\alpha_{1}, \ldots, \alpha_{m-1}, \alpha_{m}+\alpha_{m+1}\right) & \sum_{\widetilde{J} \in \mathcal{T}_{m}^{+}}(-1)^{\left(\sum_{i \in\{3, \ldots, m\} \backslash \tilde{J}} \tilde{r}_{i}\right)+m-|\widetilde{J}|} \\
= & \sum_{\widetilde{J} \in \mathcal{T}_{m}^{+} \text {s.t. } m \notin \widetilde{J}}(-1)^{\left(\sum_{i \in\{3, \ldots, m-1\} \backslash \tilde{J}} k_{i}\right)+\tilde{k}_{m}+m-|\widetilde{J}|} \\
& +\sum_{\widetilde{J} \in \mathcal{T}_{m}^{+} \text {s.t. } m \in \widetilde{J}}(-1)^{\left(\sum_{i \in\{3, \ldots, m+1\} \backslash(\tilde{J} \cup\{m+1\})} k_{i}\right)+m-|\widetilde{J}|} \\
& =\sum_{\widetilde{J} \in \mathcal{T}_{m}^{+} \text {s.t. } m \notin \widetilde{J}}(-1)^{\left(\sum_{i \in\{3, \ldots, m+1\} \backslash \tilde{J}} k_{i}\right)+m-1-|\widetilde{J}|} \\
& +\sum_{\widetilde{J} \in \mathcal{T}_{m}^{+} \text {s.t. } m \in \widetilde{J}}(-1)^{\left(\sum_{i \in\{3, \ldots, m+1\} \backslash(\tilde{J} \cup\{m+1\})} k_{i}\right)+m+1-|\widetilde{J} \cup\{m+1\}|} .
\end{aligned}
$$


On the other hand, if $\alpha_{m}-\alpha_{m+1}>0$,

$$
\begin{aligned}
& (-1)^{k_{m+1}-1} \operatorname{sgn}\left(\alpha_{m}-\alpha_{m+1}\right)^{k_{m}+k_{m+1}} \int_{\mathcal{M}\left(\alpha_{1}, \ldots, \alpha_{m-1},\left|\alpha_{m}-\alpha_{m+1}\right|\right)}\left(c_{m+1-l}^{-}\right)^{k_{m+1-l}} \\
& \cdots\left(c_{m-1}^{-}\right)^{k_{m-1}}\left(c_{m}^{-}\right)^{k_{m}+k_{m+1}-1} \\
& =(-1)^{k_{m+1}-1} \sum_{\widetilde{J} \in \mathcal{T}_{m}^{-}}(-1)^{\left(\sum_{i \in\{3, \ldots, m\} \backslash \tilde{J}^{-}} \tilde{k}_{i}\right)+m-|\widetilde{J}|} \\
& =\sum_{\widetilde{J} \in \mathcal{T}_{m}^{-} \text {s.t. } m \in \widetilde{J}}(-1)^{\left(\sum_{i \in\{3, \ldots, m\} \backslash \widetilde{J}} k_{i}\right)+\left(k_{m+1}-1\right)+m-|\widetilde{J}|} \\
& +\sum_{\widetilde{J} \in \mathcal{T}_{m}^{-} \text {s.t. } m \notin \widetilde{J}}(-1)^{\left(\sum_{i \in\{3, \ldots, m-1\} \backslash \tilde{J}} k_{i}\right)+\overbrace{k_{m}+k_{m+1}-1}^{\tilde{k}_{m}}+\left(k_{m+1}-1\right)+m-|\widetilde{J}|} \\
& =\sum_{\widetilde{J} \in \mathcal{T}_{m}^{-} \text {s.t. } m \in \widetilde{J}}(-1)^{\left(\sum_{i \in\{3, \ldots, m+1\} \backslash \widetilde{J}} k_{i}\right)+m-1-|\widetilde{J}|} \\
& +\sum_{\widetilde{J} \in \mathcal{T}_{m}^{-} \text {s.t. } m \notin \widetilde{J}}(-1)^{\left(\sum_{i \in\{3, \ldots, m+1\} \backslash(\tilde{J} \cup\{m+1\})} k_{i}\right)+m+1-|\widetilde{J} \cup\{m+1\}|},
\end{aligned}
$$

while, if $\alpha_{m}-\alpha_{m+1}<0$,

$$
\begin{aligned}
& (-1)^{k_{m+1}-1} \operatorname{sgn}\left(\alpha_{m}-\alpha_{m+1}\right)^{k_{m}+k_{m+1}} \int_{\mathcal{M}\left(\alpha_{1}, \ldots, \alpha_{m-1},\left|\alpha_{m}-\alpha_{m+1}\right|\right)}\left(c_{m+1-l}^{-}\right)^{k_{m+1-l}} \\
& \cdots\left(c_{m-1}^{-}\right)^{k_{m-1}}\left(c_{m}^{-}\right)^{k_{m}+k_{m+1}-1} \\
& =(-1)^{k_{m+1}-1+k_{m}+k_{m+1}} \sum_{\widetilde{J} \in \mathcal{T}_{m}^{-}}(-1)^{\left(\sum_{i \in\{3, \ldots, m\} \backslash \tilde{J}} \tilde{k}_{i}\right)+m-|\widetilde{J}|}
\end{aligned}
$$

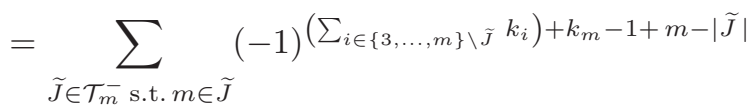

$$
\begin{aligned}
& +\sum_{\widetilde{J} \in \mathcal{T}_{m}^{-} \text {s.t. } m \notin \widetilde{J}}(-1)^{\left(\sum_{i \in\{3, \ldots, m-1\} \backslash \tilde{J}} k_{i}\right)+\overbrace{k_{m}+k_{m+1}-1}^{\tilde{k}_{m}}+k_{m}-1+m-|\widetilde{J}|} \\
& =\sum_{\widetilde{J} \in \mathcal{T}_{m}^{-} \text {s.t. } m \in \tilde{J}}(-1)^{\left(\sum_{i \in\{3, \ldots, m+1\} \backslash((\widetilde{J} \backslash\{m\}) \cup\{m+1\})} k_{i}\right)+m-1-|(\widetilde{J} \backslash\{m\}) \cup\{m+1\}|} \\
& +\sum_{\tilde{J} \in \mathcal{T}_{m}^{-} \text {s.t. } m \notin \tilde{J}}(-1)^{\left(\sum_{i \in\{3, \ldots, m+1\} \backslash(\tilde{J} \cup\{m\})} k_{i}\right)+m+1-|\widetilde{J} \cup\{m\}|},
\end{aligned}
$$

and the result follows.

Note that in the above proof we have to assume that each time we use the recursion formula we have $\alpha_{m} \neq \alpha_{m+1}$. Nevertheless, the result is still valid even if this is not the case, as long as $\alpha$ is generic. Indeed, for a generic $\alpha$ such that $\alpha_{m}=$ $\alpha_{m+1}$, we may use Remark 2.1 and take a small value of $\varepsilon>0$ for which $\mathcal{M}(\alpha)$ is diffeomorphic to $\mathcal{M}\left(\alpha_{\varepsilon}\right)$ with $\alpha:=\left(\alpha_{1}, \ldots, \alpha_{m}, \alpha_{m+1}\right)$ and $\alpha_{\varepsilon}:=\left(\alpha_{1}, \ldots, \alpha_{m}, \alpha_{m}+\right.$ $\varepsilon$ ). Note that, for $\varepsilon$ small enough, we have $\mathcal{T}_{m}^{ \pm}(\alpha)=\mathcal{T}_{m}^{ \pm}\left(\alpha_{\varepsilon}\right)$ (since $\alpha$ generic implies that $\alpha^{+}$and $\alpha^{-}$are also generic), and so the induction step still holds. 


\section{EXAMPLES}

Example 7.1. Let us consider $\mathcal{M}:=\mathcal{M}(4,3,4,3,4)$. It is a toric manifold of dimension 4 obtained by the symplectic blowing up of three points in $S^{2} \times S^{2}$. This can be seen for instance in the moment polytope depicted in Figure 1 obtained from the Hamiltonian 2-torus action given by the bending flows along the second and third diagonals. This polytope is the intersection of the rectangle $[1,7] \times[1,7]$ with the noncompact rectangular region

$$
\left\{(x, y) \in\left(\mathbb{R}_{\geq 0}\right)^{2} \mid x+y \geq 4, y \geq x-4 \text { and } y \leq x+4\right\}
$$

(cf. [7 for details on how to obtain these moment polytopes). We explicitly compute

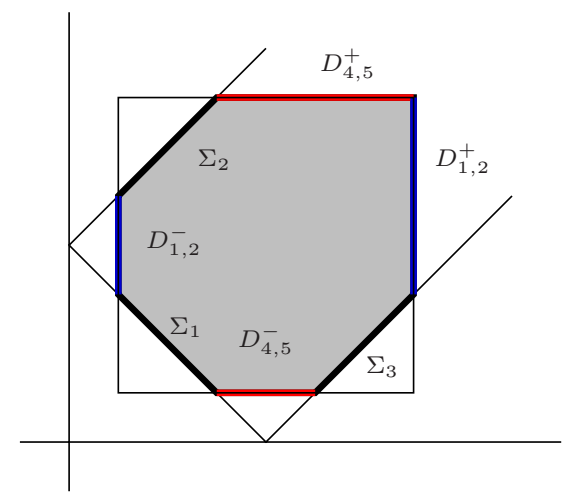

Figure 1. Moment map image for the bending action along the second and third diagonals of $\mathcal{M}(4,3,4,3,4)$.

some intersection numbers associated to this manifold. By Remark 3.1 we note that

$$
\int_{\mathcal{M}} c_{1}^{2}=\int_{\mathcal{M}} c_{3}^{2}=\int_{\mathcal{M}} c_{5}^{2} .
$$

Then, by Theorem 1.1, we have

$$
\begin{aligned}
& \int_{\mathcal{M}} c_{5}^{2}=\int_{\mathcal{M}(4,3,4,7)} c_{4}^{+} \quad-\quad \int_{\mathcal{M}(4,3,4,1)} c_{4}^{-} \\
& =\left(\int_{\mathcal{M}(4,3,11)} 1 \frac{-}{\alpha_{3}^{+}<\alpha_{4}^{+}} \int_{\mathcal{M}(4,3,3)} 1\right)-\left(\int_{\mathcal{M}(4,3,5)} 1 \frac{+}{\alpha_{3}^{-}>\alpha_{4}^{-}} \int_{\mathcal{M}(4,3,3)} 1\right) \\
& =0-1-1-1=-3 \text {. }
\end{aligned}
$$

Note that $\mathcal{M}(4,3,11)=\varnothing$ since the triple $(4,3,11)$ does not satisfy the necessary triangle inequalities $(11>4+3)$. This result can be confirmed by taking the intersections of the corresponding manifolds $D_{i, j}$. For instance, since $\alpha_{4}<\alpha_{5}$, the 
Poincaré dual of $c_{5}$ is $\left[D_{4,5}^{+}\right]-\left[D_{4,5}^{-}\right]$(cf. Proposition 4.4 ), and so

$$
\begin{aligned}
\int_{\mathcal{M}} & c_{5}^{2}=\left.\int_{\mathrm{PD}\left(c_{5}\right)} c_{5}\right|_{\mathrm{PD}\left(c_{5}\right)}=\mathrm{PD}\left(c_{5}\right) \bullet \mathrm{PD}\left(c_{5}\right) \\
& =\left(\left[D_{4,5}^{+}\right]-\left[D_{4,5}^{-}\right]\right) \bullet\left(\left[D_{4,5}^{+}\right]-\left[D_{4,5}^{-}\right]\right)=\left[D_{4,5}^{+}\right] \bullet\left[D_{4,5}^{+}\right]+\left[D_{4,5}^{-}\right] \bullet\left[D_{4,5}^{-}\right] \\
& =-1-2=-3 .
\end{aligned}
$$

The image of the manifolds $D_{4,5}^{ \pm}$by the moment map can be seen in Figure 1 and their intersection numbers can be read from the moment polytope. They are negative, and their absolute value equals the absolute value of the determinant of the $2 \times 2$-matrix formed by the inward unit normal vectors to the adjacent edges. In particular, for $D_{4,5}^{+}$and $D_{4,5}^{-}$we have

$$
D_{4,5}^{+} \bullet D_{4,5}^{+}=-\left|\operatorname{det}\left(\begin{array}{rr}
-1 & 1 \\
0 & -1
\end{array}\right)\right|=-1
$$

and

$$
D_{4,5}^{-} \bullet D_{4,5}^{-}=-\left|\operatorname{det}\left(\begin{array}{rr}
-1 & 1 \\
1 & 1
\end{array}\right)\right|=-2 .
$$

Alternatively, we could consider the circle action given by the bending flow along the second diagonal. (The image of its Hamiltonian function can be obtained by projection of the moment map polytope in Figure 1 onto the horizontal axis.) The surfaces $D_{4,5}^{+}$and $D_{4,5}^{-}$are gradient spheres for this action. The first connects an isolated fixed point of index 2 to a fixed point of index 4 (in $D_{1,2}^{+}$) (cf. [6] for the computation of these indices). Moreover, the circle rotates the sphere $D_{4,5}^{+}$with speed $k=1$ and acts linearly on the fibers over its north and south poles with weights $a=0$ and $b=-1$ respectively. Hence, by Lemma 5.4 in [11, we have $a-b=1=-e k=-e$, where $e$ is the self-intersection of $D_{4,5}^{+}$. Similarly, $D_{4,5}^{-}$is a gradient sphere connecting two fixed points of index 2. Again the circle rotates this sphere with speed 1 and acts linearly on the fibers over its north and south poles with weights 1 and -1 respectively, and so we have $2=-e$, where now $e$ is the self-intersection of $D_{4,5}^{-}$.

Similarly, by Remark 3.1 .

$$
\int_{\mathcal{M}} c_{1} c_{2}=\int_{\mathcal{M}} c_{1} c_{4}=\int_{\mathcal{M}} c_{2} c_{3}=\int_{\mathcal{M}} c_{2} c_{5}=\int_{\mathcal{M}} c_{3} c_{4}=\int_{\mathcal{M}} c_{4} c_{5},
$$

and then, by Theorem 1.1 .

$$
\begin{aligned}
& \int_{\mathcal{M}} c_{4} c_{5}=\underset{\mathcal{M}(4,3,4,7)}{\int_{\alpha_{4}<\alpha_{5}} c_{4}^{+}}+\underset{\mathcal{M}(4,3,4,1)}{\int} c_{4}^{-} \\
& =\left(\int_{\mathcal{M}(4,3,11)} 1 \frac{-}{\alpha_{3}^{+}<\alpha_{4}^{+}} \int_{\mathcal{M}(4,3,3)} 1\right)+\left(\int_{\mathcal{M}(4,3,5)} 1 \frac{+}{\alpha_{3}^{-}>\alpha_{4}^{-}} \int_{\mathcal{M}(4,3,3)} 1\right) \\
& =0-1+1+1=1 .
\end{aligned}
$$


Moreover, by Remarks 2.1 and 3.1 .

$$
\begin{aligned}
& \int_{\mathcal{M}} c_{2} c_{4}=\int_{\mathcal{M}(4,4,4,3,3+\varepsilon)} c_{4} c_{5}=\int_{\mathcal{M}(4,4,4,6)} c_{4}^{+}+\int_{\alpha_{4}<\alpha_{5}} c_{\mathcal{M}(4,4,4, \varepsilon)}^{-} \\
& =\left(\int_{\mathcal{M}(4,4,10)} 1 \frac{-}{\alpha_{3}^{+}<\alpha_{4}^{+}} \int_{\mathcal{M}(4,4,2)} 1\right)+\left(\int_{\mathcal{M}(4,4,4+\varepsilon)} 1 \underset{\alpha_{3}^{-}>\alpha_{4}^{-}}{\mathcal{M}(4,4,4-\varepsilon)} \int^{\mathcal{M}} 1\right) \\
& =0-1+1+1=1 \text {. }
\end{aligned}
$$

Again, these results can be obtained by taking the Poincaré duals. For example,

$$
\int_{\mathcal{M}} c_{2} c_{4}=\mathrm{PD}\left(c_{2}\right) \bullet \operatorname{PD}\left(c_{4}\right)=\left(D_{1,2}^{+}+D_{1,2}^{-}\right) \bullet\left(D_{4,5}^{+}+D_{4,5}^{-}\right)=D_{1,2}^{+} \bullet D_{4,5}^{+}=1 .
$$

If instead of the recursion formula (1.1) we use Theorem[1.2 we get for instance

$$
\begin{aligned}
\int_{\mathcal{M}} c_{5}^{2} & =\sum_{J \in \mathcal{T}(4,3,4,3,4)}(-1)^{\left(\sum_{i \in I \backslash J} k_{i}\right)+m-|J|} \\
& =(-1)^{k_{5}+3}+(-1)^{k_{4}+3}+(-1)^{k_{3}+3}=-3,
\end{aligned}
$$

since $\mathcal{T}(4,3,4,3,4)=\{\{3,4\},\{3,5\},\{4,5\}\}, k_{5}=2, k_{4}=k_{3}=0$ and $m-|J|=3$. Moreover,

$$
\begin{aligned}
\int_{\mathcal{M}} c_{2}^{2} & =\int_{\mathcal{M}(4,4,4,3,3)} c_{5}^{2}=\int_{\mathcal{M}(4,4,4,3,3+\varepsilon)} c_{5}^{2}=\sum_{\substack{J \in \mathcal{T}(4,4,4,3,3) \\
=\mathcal{T}(4,4,4,3,3+\varepsilon)}}(-1)^{\left(\sum_{i \in I \backslash J} k_{i}\right)+m-|J|} \\
& =(-1)^{k_{5}+3}+(-1)^{k_{4}+3}+(-1)^{k_{3}+3}=-3,
\end{aligned}
$$

since $\mathcal{T}(4,4,4,3,3)=\{\{3,4\},\{3,5\},\{4,5\}\}, k_{5}=2$ and $k_{4}=k_{3}=0$.

Using similar arguments we can easily obtain the complete list of intersection numbers for $\mathcal{M}$ (cf. Table 1).

TABLE 1. Intersection numbers for $\mathcal{M}=\mathcal{M}(4,3,4,3,4)$.

\begin{tabular}{||c|c||}
\hline $\int_{\mathcal{M}} c_{i}^{2}=-3$ & $i=1, \ldots, 5$ \\
\hline $\int_{\mathcal{M}} c_{i} c_{j}=1$ & $(i \neq j), i, j=1, \ldots, 5$ \\
\hline
\end{tabular}

It is easy to check that the classes $\left[\Sigma_{1}\right],\left[\Sigma_{2}\right]$ and $\left[\Sigma_{3}\right]$ of the exceptional divisors of the blow ups of $S^{2} \times S^{2}$ satisfy

$$
\begin{aligned}
& {\left[\Sigma_{1}\right]=\frac{1}{2}\left(\left[D_{1,2}^{+}\right]-\left[D_{1,2}^{-}\right]+\left[D_{4,5}^{+}\right]-\left[D_{4,5}^{-}\right]\right),} \\
& {\left[\Sigma_{2}\right]=\frac{1}{2}\left(\left[D_{1,2}^{+}\right]-\left[D_{1,2}^{-}\right]+\left[D_{2,3}^{+}\right]-\left[D_{2,3}^{-}\right]\right),} \\
& {\left[\Sigma_{3}\right]=\frac{1}{2}\left(\left[D_{2,3}^{+}\right]-\left[D_{2,3}^{-}\right]+\left[D_{4,5}^{+}\right]-\left[D_{4,5}^{-}\right]\right),}
\end{aligned}
$$


and so

$$
\mathrm{PD}\left(\left[\Sigma_{1}\right]\right)=\frac{1}{2}\left(c_{1}+c_{5}\right), \quad \operatorname{PD}\left(\left[\Sigma_{2}\right]\right)=\frac{1}{2}\left(c_{1}+c_{3}\right) \quad \text { and } \quad \operatorname{PD}\left(\left[\Sigma_{3}\right]\right)=\frac{1}{2}\left(c_{3}+c_{5}\right) .
$$

Note incidentally that $\int_{\mathcal{M}} c_{i}^{2}$ has the same value for every $i$. This agrees with the fact proved in [8] that in any polygon space we have $c_{i}^{2}=p$ for all $i$, where $p$ is the Pontrjagin class of the principal $S O(3)$-bundle $A(\alpha) \rightarrow \mathcal{M}(\alpha)$, with

$$
A(\alpha):=\left\{\left(\vec{v}_{1}, \ldots, \vec{v}_{m}\right) \in S_{\alpha_{i}}^{2} \mid \sum \vec{v}_{i}=0\right\} .
$$

Example 7.2. Let $\alpha=(1, \ldots, 1, m-2)$. We know from [6] that $\mathcal{M}(\alpha)$ is diffeomorphic to $\mathbb{C} P^{m-3}$, so we will check our results.

For non-negative integers $k_{1}, \ldots, k_{m}$ with $k_{1}+\cdots+k_{m}=m-3$ we obtain

$$
\int_{\mathcal{M}(\alpha)} c_{1}^{k_{1}} \cdots c_{m}^{k_{m}}=(-1)^{k_{m}}
$$

Indeed, if $k_{m} \geq 1$, we have by Remark 3.1 that

$$
\int_{\mathcal{M}(\alpha)} c_{1}^{k_{1}} \cdots c_{m}^{k_{m}}=\int_{\mathcal{M}(\alpha)} c_{m-l}^{\tilde{k}_{m-l}} \cdots c_{m-1}^{\tilde{k}_{m-1}} c_{m}^{k_{m}}
$$

with $\tilde{k}_{m-l}, \ldots, \tilde{k}_{m-1} \geq 1, m-l>3$, and $\tilde{k}_{m-l}+\cdots+\tilde{k}_{m-1}+k_{m}=m-3$. Then, since $\mathcal{T}(1, \ldots, 1, m-2)=\{\{m\}\}$, Theorem 1.2 yields

$$
\int_{\mathcal{M}(\alpha)} c_{1}^{k_{1}} \cdots c_{m}^{k_{m}}=(-1)^{\tilde{k}_{m-l}+\cdots+\tilde{k}_{m-1}+m-1}=(-1)^{m-3-k_{m}+m-1}=(-1)^{k_{m}} .
$$

On the other hand, if $k_{m}=0$, again using Remark 3.1. we get

$$
\int_{\mathcal{M}(\alpha)} c_{1}^{k_{1}} \cdots c_{m}^{k_{m}}=\int_{\mathcal{M}(\alpha)} c_{m-l}^{\tilde{k}_{m-l}} \cdots c_{m-1}^{\tilde{k}_{m-1}}=\int_{\mathcal{M}(1, \ldots, 1, \underbrace{m-2}_{m-l}, 1, \ldots, 1)} c_{m-l+1}^{\tilde{k}_{m-l+1}} \cdots c_{m-1}^{\tilde{k}_{m-1}} c_{m}^{\tilde{k}_{m-l}},
$$

with $\tilde{k}_{m-l}, \ldots, \tilde{k}_{m-1} \geq 1, m-l \geq 3$ and $\tilde{k}_{m-l}+\cdots+\tilde{k}_{m-1}=m-3$. Then, since $\mathcal{T}(1, \ldots, 1, m-2,1, \ldots, 1)=\{\{m-l\}\}$, Theorem 1.2 gives

$$
\int_{\mathcal{M}(\alpha)} c_{1}^{k_{1}} \cdots c_{m}^{k_{m}}=(-1)^{\tilde{k}_{m-l+1}+\cdots+\tilde{k}_{m-1}+\tilde{k}_{m-l}+m-1}=(-1)^{m-3+m-1}=1=(-1)^{k_{m}} .
$$

We conclude that $c_{1}=\cdots=c_{m-1}=h$, where $h \in H^{2}\left(\mathbb{C} P^{m-3} ; \mathbb{Z}\right)$ is the canonical generator, while $c_{m}=-h$. This illustrates Proposition 7.3 of [8] (since, using the notation of [8], we have $V_{i}=0$ for $i=1, \ldots, m-1$ ).

Example 7.3. Let $\alpha=(\varepsilon, \ldots, \varepsilon, 1,1,1)$ with $(m-3) \varepsilon<1$. We know from 8 that $\mathcal{M}(\alpha)$ is symplectomorphic to $\prod_{i=1}^{m-3} S_{\alpha_{i}}^{2}$.

For nonnegative integers $k_{1}, \ldots, k_{m}$ with $k_{1}+\cdots+k_{m}=m-3$ we have

$$
\int_{\mathcal{M}(\alpha)} c_{1}^{k_{1}} \cdots c_{m}^{k_{m}}=0
$$


unless $k_{m-2}=k_{m-1}=k_{m}=0$ and $k_{1}=\cdots=k_{m-3}=1$, in which case we have

$$
\int_{\mathcal{M}(\alpha)} c_{1} \cdots c_{m-3}=2^{m-3} \text {. }
$$

Indeed, if $k_{m-2}, k_{m-1}, k_{m} \neq 0$, then by Remark 3.1 .

$$
\int_{\mathcal{M}(\alpha)} c_{1}^{k_{1}} \cdots c_{m}^{k_{m}}=\int_{\mathcal{M}(\alpha)} c_{m-l}^{\tilde{k}_{m-l}} \cdots c_{m-3}^{\tilde{k}_{m-3}} c_{m-2}^{k_{m-2}} c_{m-1}^{k_{m-1}} c_{m}^{k_{m}}
$$

with $\tilde{k}_{m-l}, \ldots, \tilde{k}_{m-3} \geq 1$ and $m-l>3$. Then, (7.1) holds since $\mathcal{T}(\varepsilon, \ldots, \varepsilon, 1,1,1)=$ $\varnothing$.

If only one of $k_{m-2}, k_{m-1}$ or $k_{m}$ is equal to zero, then, again by Remark 3.1 ,

$$
\int_{\mathcal{M}(\alpha)} c_{1}^{k_{1}} \cdots c_{m}^{k_{m}}=\int_{\mathcal{M}(\alpha)} c_{m-l}^{\tilde{k}_{m-l}} \cdots c_{m-1}^{\tilde{k}_{m-1}}
$$

with $\tilde{k}_{m-l}, \ldots, \tilde{k}_{m-1} \geq 1$ and $m-l \geq 3$, and so

$$
\left.\int_{\mathcal{M}(\alpha)} c_{1}^{k_{1}} \cdots c_{m}^{k_{m}}=\int_{\mathcal{M}(\varepsilon, \ldots, \varepsilon, \underbrace{1}_{m-l}} c_{m-l+1}^{\tilde{k}_{m-l+1}} \cdots c_{m-1}^{\tilde{k}_{m-1}} c_{m}^{\tilde{k}_{m-l}}=0, \varepsilon, 1,1, \varepsilon\right)
$$

since, for $m-l>3, \mathcal{T}(\varepsilon, \ldots, \varepsilon, \underbrace{1}_{m-l}, \varepsilon, \ldots, \varepsilon, 1,1, \varepsilon)=\varnothing$.

If two of $k_{m-2}, k_{m-1}$ or $k_{m}$ are zero, then by Remark 3.1 .

$$
\int_{\mathcal{M}(\alpha)} c_{1}^{k_{1}} \cdots c_{m}^{k_{m}}=\int_{\mathcal{M}(\alpha)} c_{m-l}^{\tilde{k}_{m-l}} \cdots c_{m-2}^{\tilde{k}_{m-2}}
$$

with $\tilde{k}_{m-l}, \ldots, \tilde{k}_{m-2} \geq 1$ and $m-l \geq 2$, and so

$$
\int_{\mathcal{M}(\alpha)} c_{1}^{k_{1}} \cdots c_{m}^{k_{m}}=\int_{\mathcal{M}(\varepsilon, \ldots, \varepsilon, \underbrace{1}_{m-l}, 1, \varepsilon, \ldots, \varepsilon, 1, \varepsilon, \varepsilon)} c_{m-l+2}^{\tilde{k}_{m-l+2}} \cdots c_{m-2}^{\tilde{k}_{m-2}} c_{m-1}^{\tilde{k}_{m-l}} c_{m}^{\tilde{k}_{m-l+1}}=0
$$

since both $\mathcal{T}(\varepsilon, 1,1, \varepsilon \ldots, \varepsilon, 1, \varepsilon, \varepsilon)$ and $\mathcal{T}(\varepsilon, \varepsilon, \ldots, \varepsilon, \underbrace{1}_{m-l}, 1, \varepsilon, \ldots, \varepsilon, 1, \varepsilon, \varepsilon)$ (respectively the cases $m-l=2$ and $m-l>2$ ) are empty.

If $k_{m-2}=k_{m-1}=k_{m}=0$, then

$$
\int_{\mathcal{M}(\alpha)} c_{1}^{k_{1}} \cdots c_{m}^{k_{m}}=\int_{\mathcal{M}(\alpha)} c_{m-l}^{\tilde{k}_{m-l}} \cdots c_{m-3}^{\tilde{k}_{m-3}}
$$

with $\tilde{k}_{m-l}, \ldots, \tilde{k}_{m-3} \geq 1$ and $m-l \geq 1$. If $m-l \geq 2$, then we do as before and again obtain $\mathcal{T}=\varnothing$, implying that (7.1) holds. If, however, $m-l=1$, then necessarily $k_{1}=\cdots=k_{m-3}=1$ and then

$$
\int_{\mathcal{M}(\alpha)} c_{1}^{k_{1}} \cdots c_{m}^{k_{m}}=\int_{\mathcal{M}(\alpha)} c_{1} \cdots c_{m-3}=\int_{\mathcal{M}(1,1,1, \varepsilon, \ldots, \varepsilon)} c_{4} \cdots c_{m} .
$$


Now $\mathcal{T}(1,1,1, \varepsilon, \ldots, \varepsilon)=\{J \subset I:=\{3, \ldots, m\} \mid 3 \in J\}$, and so Theorem 1.2 yields

$$
\begin{aligned}
\int_{\mathcal{M}(1,1,1, \varepsilon, \ldots, \varepsilon)} c_{4} \cdots c_{m} & =\sum_{J \in \mathcal{T}}(-1)^{\left(\sum_{i \in I \backslash J} 1\right)+m-|J|}=\sum_{J \in \mathcal{T}}(-1)^{|I|-|J|+m-|J|} \\
& =\sum_{J \in \mathcal{T}}(-1)^{m-2+m}=|\mathcal{T}|=\sum_{i=0}^{m-3}\left(\begin{array}{c}
m-3 \\
i
\end{array}\right)=2^{m-3} .
\end{aligned}
$$

For example, if $m=5, \mathcal{M}(\varepsilon, \varepsilon, 1,1,1)$ is $S^{2} \times S^{2}$ and the Poincaré duals of $c_{1}$ and $c_{2}$ are $2\left[\{\mathrm{pt}\} \times S^{2}\right]$ and $2\left[S^{2} \times\{\mathrm{pt}\}\right]$. Hence, $\int_{\mathcal{M}(\alpha)} c_{1} c_{2}=4$, while $\int_{\mathcal{M}(\alpha)} c_{1}^{2}=$ $\int_{\mathcal{M}(\alpha)} c_{2}^{2}=0$. Moreover, $c_{3}=c_{4}=c_{5}=0$.

In general, for $\mathcal{M}(\varepsilon, \ldots, \varepsilon, 1,1,1)=\prod_{j=1}^{m-3} S_{\alpha_{j}}^{2}$, we have

$$
c_{i}=\operatorname{PD}\left(2\left[\prod_{j \neq i} S_{\alpha_{j}}^{2}\right]\right) \quad \text { for } \quad i=1, \ldots, m-3,
$$

while $c_{m-2}=c_{m-1}=c_{m}=0$.

\section{Equilateral POLyGon SPACES}

Here we study the equilateral case corresponding to $\alpha_{i}=1$ for all $i$. Since we want $\alpha$ to be generic we need $m$ to be odd. The intersection numbers for these spaces were computed by Kamiyama and Tezuka in [17. Their result states the following.

Theorem 8.1 (Kamiyama-Tezuka). Let $\left(d_{1}, \ldots, d_{m}\right)$ be a sequence of nonnegative integers with $\sum d_{i}=m-3$. Let $\beta_{i}, \varepsilon_{i}$ be such that $d_{i}=2 \beta_{i}+\varepsilon_{i}$, where $\varepsilon_{i}=0$ or 1. Then, defining

$$
\rho_{m, 2 k}:=(-1)^{k} \frac{\left(\begin{array}{c}
\frac{m-3}{2} \\
k
\end{array}\right)\left(\begin{array}{c}
m-2 \\
\frac{m-1}{2}
\end{array}\right)}{\left(\begin{array}{c}
m-2 \\
2 k+1
\end{array}\right)}
$$

we have

(1) if $\beta_{i}=0$ for $1 \leq i \leq m$, then $\int c_{1}^{d_{1}} \cdots c_{m}^{d_{m}}=\rho_{m, 0}$;

(2) if $\beta_{i} \neq 0$ for some $i$, then $\int c_{1}^{d_{1}} \cdots c_{m}^{d_{m}}=\rho_{m, 2 k}$, with $k=\beta_{1}+\cdots+\beta_{m}$.

We will see how to obtain this result using Theorem 1.2 .

Proof. 1. First we see that if $\beta_{i}=0$ for $1 \leq i \leq m$, then, since $\sum d_{i}=m-3$, we have

$$
\int_{\mathcal{M}_{m}} c_{1}^{d_{1}} \cdots c_{m}^{d_{m}}=\int c_{4} \cdots c_{m}
$$


Moreover, $\mathcal{T}=\left\{J \subset\{3, \ldots, m\}|| J \mid=\frac{m-1}{2}\right\}$, and so

$$
\begin{aligned}
\int_{\mathcal{M}_{m}} c_{4} \cdots c_{m} & =\sum_{J \in \mathcal{T}}(-1)^{|(I \backslash\{3\}) \backslash J|+m-|J|}=\sum_{J \in \mathcal{T}}(-1)^{|(I \backslash\{3\}) \backslash J|+\frac{m+1}{2}} \\
= & \sum_{J \in \mathcal{T} \text { s.t. } 3 \in J}(-1)^{\frac{m-3}{2}+\frac{m+1}{2}}+\sum_{J \in \mathcal{T} \text { s.t. } 3 \notin J}(-1)^{\frac{m-3}{2}-1+\frac{m+1}{2}} \\
= & \left(\begin{array}{c}
m-3 \\
\frac{m-3}{2}
\end{array}\right)(-1)^{m-1}+\left(\begin{array}{c}
m-3 \\
\frac{m-1}{2}
\end{array}\right)(-1)^{m-2} \\
= & (-1)^{m-1}\left\{\left(\begin{array}{c}
m-3 \\
\frac{m-3}{2}
\end{array}\right)-\left(\begin{array}{c}
m-3 \\
\frac{m-1}{2}
\end{array}\right)\right\}=\frac{(m-3) !}{\left(\frac{m-1}{2}\right) !\left(\frac{m-3}{2}\right) !}=\rho_{m, 0} .
\end{aligned}
$$

2. Let us now assume that $\beta_{j} \neq 0$ for some $j$. Since $d_{1}+\cdots+d_{m}=m-3$ we must have $d_{j}=0$ for some $j$. Then, using the fact that $c_{1}^{2}=c_{2}^{2}=\cdots=c_{m}^{2}$ (cf. [8, 1, 12]) as well as Remark 3.1, we have

$$
\int_{\mathcal{M}_{m}} c_{1}^{d_{1}} \cdots c_{m}^{d_{m}}=\int_{\mathcal{M}_{m}} c_{2 k+3} c_{2 k+4} \cdots c_{m-1} c_{m}^{2 k}
$$

with $k=\beta_{1}+\cdots+\beta_{m}$. Now we have to consider two cases.

If $2 k=m-3$, then, since $\mathcal{T}=\left\{J \subset I:=\{3, \ldots, m\}|| J \mid=\frac{m-1}{2}\right\}$, we have

$$
\begin{gathered}
\int_{\mathcal{M}_{m}} c_{m}^{2 k}=\sum_{J \in \mathcal{T}}(-1)^{\sum_{i \in(I \backslash J)} k_{i}+m-|J|}=(-1)^{\frac{m+1}{2}} \sum_{J \in \mathcal{T}}(-1)^{\sum_{i \in I \backslash J} k_{i}} \\
=(-1)^{\frac{m+1}{2}}|\mathcal{T}|=(-1)^{\frac{m+1}{2}}\left(\begin{array}{c}
m-2 \\
\frac{m-1}{2}
\end{array}\right)=\rho_{m, m-3},
\end{gathered}
$$

where we used the fact that all the exponents $k_{i}$ in (8.1) are even $\left(k_{i}=0\right.$ if $i \neq m$ and $\left.k_{m}=2 k\right)$.

If, on the other hand, $2 k \neq m-3$, let us consider the set $A:=\{2 k+3, \ldots, m-$ $1\} \subset I$. Then

$$
\begin{aligned}
\int_{\mathcal{M}_{m}} c_{2 k+3} c_{2 k+4} \cdots c_{m-1} c_{m}^{2 k} & =\sum_{J \in \mathcal{T}}(-1)^{\sum_{i \in(I \backslash J)} k_{i}+m-|J|} \\
& =(-1)^{\frac{m+1}{2}} \sum_{J \in \mathcal{T}}(-1)^{|(I \backslash J) \cap A|} .
\end{aligned}
$$

Note that $|I \backslash J|=\frac{m-3}{2},|I \backslash A|=2 k+1,|A|=m-2 k-3$ and that if there exist $j$ elements in $(I \backslash J) \cap(I \backslash A)$, then there exist $\frac{m-3}{2}-j$ elements in $(I \backslash J) \cap A$. Hence,

$$
\begin{aligned}
\int_{\mathcal{M}_{m}} & c_{2 k+3} c_{2 k+4} \cdots c_{m-1} c_{m}^{2 k} \\
= & (-1)^{\frac{m+1}{2}} \sum_{j=0}^{2 k+1}\left(\begin{array}{c}
2 k+1 \\
j
\end{array}\right)\left(\begin{array}{c}
m-2 k-3 \\
\frac{m-3}{2}-j
\end{array}\right)(-1)^{\frac{m-3}{2}-j} \\
= & \sum_{j=0}^{2 k+1}(-1)^{j}\left(\begin{array}{c}
2 k+1 \\
j
\end{array}\right)\left(\begin{array}{c}
m-2 k-3 \\
\frac{m-3}{2}-j
\end{array}\right) .
\end{aligned}
$$


The result now follows from the following combinatorial identity:

$$
\sum_{j=0}^{2 k+1}(-1)^{j+k} \frac{\left(\begin{array}{c}
2 k+1 \\
j
\end{array}\right)\left(\begin{array}{c}
m-2 k-3 \\
\frac{m-3}{2}-j
\end{array}\right)\left(\begin{array}{c}
m-2 \\
2 k+1
\end{array}\right)}{\left(\begin{array}{c}
m-2 \\
\frac{m-1}{2}
\end{array}\right)}=\left(\begin{array}{c}
\frac{m-3}{2} \\
k
\end{array}\right),
$$

since then

$$
\int_{\mathcal{M}_{m}} c_{2 k+3} c_{2 k+4} \cdots c_{m-1} c_{m}^{2 k}=(-1)^{k} \frac{\left(\begin{array}{c}
\frac{m-3}{2} \\
k
\end{array}\right)\left(\begin{array}{c}
m-2 \\
\frac{m-1}{2}
\end{array}\right)}{\left(\begin{array}{c}
m-2 \\
2 k+1
\end{array}\right)}=\rho_{2,2 k} .
$$

Hence to finish the proof we just have to show (8.2). For that we see that

$$
\begin{aligned}
& \sum_{j=0}^{2 k+1}(-1)^{j+k} \frac{\left(\begin{array}{c}
2 k+1 \\
j
\end{array}\right)\left(\begin{array}{c}
m-2 k-3 \\
\frac{m-3}{2}-j
\end{array}\right)\left(\begin{array}{c}
m-2 \\
2 k+1
\end{array}\right)}{\left(\begin{array}{c}
m-2 \\
\frac{m-1}{2}
\end{array}\right)} \\
& =\sum_{j=0}^{2 k+1}(-1)^{j+k} \frac{\left(\frac{m-1}{2}\right) !\left(\frac{m-3}{2}\right) !}{j !(2 k+1-j) !\left(\frac{m-3}{2}-j\right) !\left(\frac{m-3}{2}-2 k+j\right) !} \\
& =\sum_{j=0}^{2 k+1}(-1)^{j+k}\left(\begin{array}{c}
\frac{m-3}{2} \\
j
\end{array}\right)\left(\begin{array}{c}
\frac{m-1}{2} \\
2 k+1-j
\end{array}\right)=\left(\begin{array}{c}
\frac{m-3}{2} \\
k
\end{array}\right),
\end{aligned}
$$

where the last equality was obtained from the combinatorial Lemma 8.2 stated below, using $a=\frac{m-3}{2}$ and $b=2 k+1$.

Lemma 8.2. For any odd integer $b$ we have

$$
\sum_{j=0}^{b}(-1)^{j}\left(\begin{array}{c}
a \\
j
\end{array}\right)\left(\begin{array}{c}
a+1 \\
b-j
\end{array}\right)=(-1)^{\frac{b-1}{2}}\left(\begin{array}{c}
a \\
\frac{b-1}{2}
\end{array}\right) .
$$

Proof of Lemma 8.2. This binomial identity can be easily proved using a generating function (cf. 4] for a definition of generating function in this context). Indeed, since

$$
(z-1)^{a}(z+1)^{a+1}=\left(z^{2}-1\right)^{a}(1+z)
$$

and

$$
\begin{aligned}
& (z-1)^{a}=\sum_{j \geq 0}(-1)^{a+j}\left(\begin{array}{c}
a \\
j
\end{array}\right) z^{j}, \quad(z+1)^{a}=\sum_{j \geq 0}\left(\begin{array}{c}
a \\
j
\end{array}\right) z^{j}, \\
& \left(z^{2}-1\right)^{a}(1+z)=\sum_{j \geq 0}(-1)^{a+j}\left(\begin{array}{c}
a \\
j
\end{array}\right) z^{2 j}+\sum_{j \geq 0}(-1)^{a+j}\left(\begin{array}{c}
a \\
j
\end{array}\right) z^{2 j+1},
\end{aligned}
$$

the result follows from equating coefficients of $z^{b}$ in (8.3) for an odd $b$.

Example 8.3. If $m=5$ we obtain

$$
\int_{\mathcal{M}_{5}} c_{i}^{2}=\rho_{5,2}=-3 \text { and } \int_{\mathcal{M}_{5}} c_{i} c_{j}=\rho_{5,0}=1, \text { if } i \neq j .
$$

Incidentally, note that these values are equal to the ones obtained in Example 7.1 This is not surprising since $\mathcal{M}_{5}$ is diffeomorphic to $\mathcal{M}(4,3,4,3,4)$. Indeed, by 
Remark 2.1] $\mathcal{M}_{5}$ is diffeomorphic to $\mathcal{M}\left(\alpha_{\varepsilon}\right)$ with $\alpha_{\varepsilon}=(1+\varepsilon, 1,1,1,1+\varepsilon)$ for small values of $\varepsilon$, and $\mathcal{M}\left(\alpha_{\varepsilon}\right)$ is a toric manifold whose moment polytope has 7 edges just like the one for $\mathcal{M}(4,3,4,3,4)$ depicted in Figure 1 (the three spaces are all diffeomorphic to $\left.\left(S^{2} \times S^{2}\right) \# 3 \overline{\mathbb{C} P^{2}}\right)$.

The space of equilateral polygons $\mathcal{M}_{m}$ admits a natural action of the symmetric group Sym $_{m}$. Moreover, the quotient $\mathcal{M}_{m} / S y m_{m}$ can be seen as a compactification of the moduli space of $m$ unordered points in $\mathbb{C} P^{1}$ as well as the space of $m$-times punctured genus zero algebraic curves. The cohomology ring of this space was computed by Brion [1, Klyachko 12 and Hausmann and Knutson 8 . Since

$$
H^{*}\left(\mathcal{M}_{m} / \text { Sym }_{m} ; \mathbb{Q}\right) \cong H^{*}\left(\mathcal{M}_{m} ; \mathbb{Q}\right)^{\text {Sym }_{m}}
$$

can be identified with the invariant part of $H^{*}\left(\mathcal{M}_{m} ; \mathbb{Q}\right)$, and $H^{*}\left(\mathcal{M}_{m} ; \mathbb{Q}\right)$ is generated in degree 2, we just have to study the action of $S y m_{m}$ on $H^{2}\left(\mathcal{M}_{m}\right)$. Hausmann and Knutson 8 proved that this $\operatorname{Sym}_{m}$-invariant part of $H^{*}\left(\mathcal{M}_{m} ; \mathbb{Q}\right)$ is generated by $\sigma_{1}$, the first invariant symmetric polynomial in the classes $c_{i}$, and by $c_{m}^{2}$ (or any other $c_{i}^{2}$ since they are all equal to the Pontrjagin class of the principal $S O(3)$-bundle $A_{m} \rightarrow \mathcal{M}_{m}$ with $\left.A_{m}:=\left\{\left(\vec{v}_{1}, \ldots, \vec{v}_{m}\right) \in\left(\mathbb{R}^{3}\right)^{m}\left|\sum_{i=1}^{m} \vec{v}_{i}=0,\right| \vec{v}_{1}|=\cdots=| \vec{v}_{m} \mid\right\}\right)$, a generator of degree 4 , with no relations up to degree $m-3$. The intersection numbers $\sigma_{1}^{k} \cdot c_{m}^{m-3-k}$ for an even $k$ can be obtained from Theorem 8.1 First we see that

$$
\sigma_{1}^{k}=\sum_{k_{1}+\cdots+k_{m}=k} \frac{k !}{k_{1} ! \cdots k_{m} !} c_{1}^{k_{1}} \cdots c_{m}^{k_{m}} .
$$

Then

$$
\begin{aligned}
\int_{\mathcal{M}_{m} / \text { Sym }_{m}} \sigma_{1}^{k} \cdot c_{m}^{m-3-k} & =\sum_{k_{1}+\cdots+k_{m}=k} \frac{k !}{k_{1} ! \cdots k_{m} !} \int_{\mathcal{M}_{m}} c_{1}^{k_{1}} \cdots c_{m-1}^{k_{m-1}} c_{m}^{k_{m}+m-3-k} \\
& =\sum_{k_{1}+\cdots+k_{m}=k} \frac{k !}{k_{1} ! \cdots k_{m} !} \rho_{m, 2\left(\beta_{1}+\cdots+\beta_{m-1}+\tilde{\beta}_{m}\right)},
\end{aligned}
$$

where the $\beta_{i}$ are such that $k_{i}=2 \beta_{i}+\varepsilon_{i}, \varepsilon_{i}=0$ or 1 and $\tilde{\beta}_{m}=\beta_{m}+\frac{m-3-k}{2}$. Hence,

$$
2\left(\beta_{1}+\cdots+\beta_{m-1}+\tilde{\beta}_{m}\right)=m-3-\text { number of odd } k_{i} \text { 's }
$$

and so, since the number of odd $k_{i}$ 's must be even ( $k$ is even), we have

$$
\int_{\mathcal{M}_{m} / \text { Sym }_{m}} \sigma_{1}^{k} \cdot c_{m}^{m-3-k}=\sum_{j=0}^{\frac{k}{2}} \sum_{k_{1}+\cdots+k_{m}=k \text { s.t. }} \frac{k !}{k_{1} ! \cdots k_{m} !} \rho_{m, m-3-2 j},
$$

$2 j$ of the $k_{i}$ 's are odd

and we obtain Proposition 1.3 .

\section{Moduli space of flat Connections}

Polygon spaces can be identified with moduli spaces of flat $S U(2)$-connections on the $m$-punctured sphere. In this section we compare the existing formulas for cohomology intersection pairings in the context of moduli spaces of flat connections with our explicit expression of Theorem 1.2

Let us consider closed polygons in $S^{3}$ with vertices $v_{1}, \ldots, v_{n}$ joined by edges $e_{1}, \ldots, e_{n}$, where each $e_{i}$ is the geodesic arc from $v_{i}$ to $v_{i+1}$. The length $\alpha_{i}$ of an edge is then the length of this geodesic arc. Let us denote by $\mathcal{M}(\alpha)^{S^{3}}$ the moduli 
space of closed polygons in $S^{3}$ with side-lengths $\alpha$ modulo orientation-preserving isometries, that is, modulo $S O(4)$. This space can be identified with the moduli space of flat $S U(2)$-connections on a punctured sphere with fixed holonomies around the punctures. Indeed, let us denote by $\mathcal{M}\left(S_{m}, \alpha\right)$ the moduli space of flat $S U(2)$ connections on the $m$-punctured sphere $S_{m}:=S^{2} \backslash\left\{p_{1}, \ldots, p_{m}\right\}$ modulo gauge equivalence such that the holonomy around $p_{i}$ is conjugate to

$$
A_{\alpha_{i}}:=\left(\begin{array}{cc}
e^{i \pi \alpha_{i}} & 0 \\
0 & e^{-i \pi \alpha_{i}}
\end{array}\right)
$$

Then,

$$
\mathcal{M}\left(S_{m}, \alpha\right) \cong \mathcal{R}(m, \alpha) / S U(2)
$$

where

$\mathcal{R}(m, \alpha)=\left\{\left(g_{1}, \ldots, g_{m}\right) \in S U(2)^{m} \mid g_{1} \cdots g_{m}=I d, \operatorname{tr} g_{i}=2 \cos \pi \alpha_{i}, i=1, \ldots, m\right\}$

is the space of representations from $\pi_{1}\left(S_{m}\right)$ to $S U(2)$ such that the image of the loop around the puncture $p_{i}$ is conjugate to $A_{\alpha_{i}}$ and $S U(2)$ acts diagonally.

Moreover, $\mathcal{R}(m, \alpha)$ can be identified with the space $\mathcal{P}(\alpha)^{S^{3}, 0}$ of based polygons in $S^{3}$ that have the first vertex fixed $v_{1}=I d \in S U(2) \cong S^{3}$. Each element $\left(g_{1}, \ldots, g_{m}\right) \in \mathcal{R}(m, \alpha)$ is identified with the based polygon that has vertices

$$
v_{1}=I d, \quad v_{2}=g_{1}, \quad v_{3}=g_{1} g_{2}, \quad \cdots \quad v_{i}=g_{1} g_{2} \cdots g_{i-1}, \quad i=1, \ldots, m .
$$

Then,

$$
\mathcal{M}\left(S_{m}, \alpha\right) \cong \mathcal{R}(m, \alpha) / S U(2) \cong \mathcal{P}(\alpha)^{S^{3}, 0} / S O(3) \cong \mathcal{M}(\alpha)^{S^{3}} / S O(4)
$$

A formula for the symplectic volume of the space $\mathcal{M}\left(S_{m}, \alpha\right)$ was first obtained by Witten in [25] and proved rigorously by Jeffrey and Weitsman in [10]. It states the following.

Theorem 9.1 (Witten, Jeffrey-Weitsman).

$$
\operatorname{Vol}\left(\mathcal{M}\left(S_{m}, \alpha\right)\right)=\frac{4}{\pi^{m-2}} \sum_{k=1}^{\infty} \frac{\prod_{i=1}^{m} \sin \left(k \pi \alpha_{i}\right)}{k^{m-2}} .
$$

Later in [21] $\mathrm{Vu}$ The Khoi obtained a closed form expression for this volume in terms of Bernoulli polynomials. Noting that the moduli spaces of polygons in $S^{3}$ and in the Euclidean space $\mathbb{R}^{3}$ with the same side lengths are symplectomorphic provided that the $\alpha_{i}$ are sufficiently small (cf. Theorem 6.6 in [9] and [16]) and that multiplying $\alpha$ by a scalar $\lambda>0$ we get $\operatorname{vol}(\mathcal{M}(\lambda \alpha))=\lambda^{m-3} \operatorname{vol}(\mathcal{M}(\alpha))$, he deduces an expression for the volume of $\mathcal{M}(\alpha)$.

Proposition 9.2 (Vu The Khoi). The symplectic volume of the moduli space $\mathcal{M}(\alpha)$ for $\alpha=\left(\alpha_{1}, \ldots, \alpha_{m}\right)$ is given by

$$
\operatorname{Vol}(\mathcal{M}(\alpha))=-\frac{1}{4(m-3) !} \sum_{R \subset\{1, \ldots, m\}}(-1)^{|R|} \operatorname{sgn}\left(\sum_{i \in R} \alpha_{i}-\sum_{i \notin R} \alpha_{i}\right)\left(\sum_{i \in R} \alpha_{i}-\sum_{i \notin R} \alpha_{i}\right)^{m-3}
$$

if $m$ is even and by

$$
\operatorname{Vol}(\mathcal{M}(\alpha))=-\frac{1}{2(m-3) !} \sum_{R \subset\{1, \ldots, m\} \text { s.t. }|R| \text { odd }} \operatorname{sgn}\left(\sum_{i \in R} \alpha_{i}-\sum_{i \notin R} \alpha_{i}\right)\left(\sum_{i \in R} \alpha_{i}-\sum_{i \notin R} \alpha_{i}\right)^{m-3}
$$

if $m$ is odd. 
A similar expression was obtained independently by Mandini in [19] using localization theorems in equivariant cohomology and an equivariant integration formula for symplectic quotients by nonabelian groups.

Using the Witten-Jeffrey-Weitsman expression for the volume of $\mathcal{M}\left(S_{m}, \alpha\right)$, Yoshida obtains in 27] a generating function for cohomology intersection pairings of the moduli space of flat connections.

Theorem 9.3 (Yoshida). Let $t_{1}, \ldots, t_{m} \in \mathbb{R}$. Then,

$$
\sum_{k_{1}, \ldots, k_{m} \geq 0} \frac{t_{1}^{k_{1}}}{k_{1} !} \cdots \frac{t_{m}^{k_{1}}}{k_{m} !} \int_{\mathcal{M}\left(S_{m}, \alpha\right)} c_{1}^{k_{1}} \cdots c_{m}^{k_{m}}=\frac{4}{\pi^{m-2}} \sum_{l=1}^{\infty} \frac{\prod_{j=1}^{m} \sin \left(\pi l\left(\alpha_{j}+x_{j}\right)\right)}{l^{m-2}},
$$

where $\sum_{i=1}^{m} k_{i}=m-3$.

The cohomology intersection pairings $\int_{\mathcal{M}\left(S_{m}, \alpha\right)} c_{1}^{k_{1}} \cdots c_{1}^{k_{m}}$ can then be obtained from the above expression (9.1) for the volume by taking the appropriate derivatives

$$
\int_{\mathcal{M}\left(S_{m}, \alpha\right)} c_{1}^{k_{1}} \cdots c_{m}^{k_{m}}=\left.\frac{\partial^{k_{1}}}{\partial t_{1}^{k_{1}}} \cdots \frac{\partial^{k_{m}}}{\partial t_{m}^{k_{m}}} \operatorname{Vol}\left(\mathcal{M}\left(S_{m}, \alpha+t\right)\right)\right|_{t=0} .
$$

Using this formula and the identification of $\mathcal{M}\left(S_{m}, \alpha\right)$ with $\mathcal{M}(\alpha)$ for small values of $\alpha$ one obtains a formula for intersection pairings in $\mathcal{M}(\alpha)$ by taking derivatives of the expressions in Proposition 9.2.

\section{Theorem 9.4.}

$$
\int_{\mathcal{M}\left(S_{m}, \alpha\right)} c_{1}^{k_{1}} \cdots c_{m}^{k_{m}}=\frac{1}{4} \sum_{R \subset\{1, \ldots, m\}}(-1)^{|R|+1+\sum_{i \notin R} k_{i}} \operatorname{sgn}\left(\sum_{i \in R} \alpha_{i}-\sum_{i \notin R} \alpha_{i}\right)
$$

if $m$ is even and

$$
\int_{\mathcal{M}\left(S_{m}, \alpha\right)} c_{1}^{k_{1}} \cdots c_{m}^{k_{m}}=\frac{1}{2} \sum_{R \subset\{1, \ldots, m\} \text { s.t. }|R| \text { odd }}(-1)^{1+\sum_{i \notin R} k_{i}} \operatorname{sgn}\left(\sum_{i \in R} \alpha_{i}-\sum_{i \notin R} \alpha_{i}\right)
$$

if $m$ is odd.

We will now see how this formula is equivalent to our explicit expression of Theorem 1.2.

Proof. Let us assume without loss of generality that $\alpha_{1}>\alpha_{2}$ and let $I=\{3, \ldots, m\}$. Notice that

$$
\begin{aligned}
& \{R \subset\{1, \ldots, m\}\}=\{R \subset I \mid R \in \mathcal{T}(\alpha)\} \cup\{R \subset I \mid R \notin \mathcal{T}(\alpha)\} \\
& \cup\{R=\{1,2\} \cup J \mid J \subset I\} \cup\{R=\{1\} \cup J \mid J \subset I\} \cup\{R=\{2\} \cup J \mid J \subset I\} .
\end{aligned}
$$

Let us denote by $S_{R}$ the difference

$$
S_{R}:=\sum_{i \in R} \alpha_{i}-\sum_{i \notin R} \alpha_{i}
$$

and by $l_{J}$ the sum

$$
l_{J}:=\sum_{i=3}^{m}(-1)^{\chi_{I \backslash J}(i)} \alpha_{i},
$$

whenever $J \subset I$. 
If $R \subset I$ and $R \in \mathcal{T}(\alpha)$, then $S_{R}=l_{R}-\alpha_{1}-\alpha_{2} \leq 0$ since for triangular sets $l_{R} \leq \alpha_{1}+\alpha_{2}$. If $R \subset I$ is not triangular, then, if $l_{R} \leq 0$ or $0<l_{R}<\alpha_{1}-\alpha_{2}$, we have $S_{R}<0$. If, however, $l_{R}>\alpha_{1}+\alpha_{2}$, then $S_{R}>0$.

If $R=\{1,2\} \cup J$ with $J \subset I$ we have $|R|=|J|+2$ and $S_{R}=l_{J}+\alpha_{1}+\alpha_{2}$. If in addition $J \in \mathcal{T}(\alpha)$, then $S_{R}>0$. If, on the other hand, $J \notin \mathcal{T}(\alpha)$, then $S_{R}>0$ whenever $0>l_{J}>-\left(\alpha_{1}+\alpha_{2}\right)$ or $l_{J}>0$ and negative otherwise.

If $R=\{1\} \cup J$ with $J \subset I$, then $|R|=|J|+1$ and $S_{R}=l_{J}+\alpha_{1}-\alpha_{2}$. If $J \in \mathcal{T}(\alpha)$, then $S_{R}>0$. If, however, $J \notin \mathcal{T}(\alpha)$, then $S_{R}>0$ whenever $0>l_{J}>-\left(\alpha_{1}-\alpha_{2}\right)$ or $l_{J}>0$ and negative otherwise.

Similarly, if $R=\{2\} \cup J$ with $J \subset I$ we have $|R|=|J|+1$ and $S_{R}=l_{J}+\alpha_{2}-\alpha_{1}$. If in addition $J \in \mathcal{T}(\alpha)$, then $S_{R}>0$ (since by the triangular inequalities $l_{J} \geq$ $\left.\left|\alpha_{2}-\alpha_{1}\right|\right)$. If, on the other hand, $J \notin \mathcal{T}(\alpha)$, then $S_{R}>0$ if $l_{J}>\alpha_{1}+\alpha_{2}$ and negative otherwise.

Let us assume first that $m$ is even. Putting the above information together, the RHS of (9.2) is equal to

$$
\begin{aligned}
& \frac{1}{4} \sum_{J \in \mathcal{T}(\alpha)}(-1)^{|J|+\sum_{i \in I \backslash J} k_{i}}\left((-1)^{k_{1}+k_{2}}-1+(-1)^{k_{2}}+(-1)^{k_{1}}\right) \\
& +\sum_{J \notin \mathcal{T}(\alpha) \text { s.t. } l_{J}>\alpha_{1}+\alpha_{2}}(-1)^{|J|+\sum_{i \in I \backslash J} k_{i}}\left((-1)^{1+k_{1}+k_{2}}-1+(-1)^{k_{2}}+(-1)^{k_{1}}\right) \\
& +\sum_{J \notin \mathcal{T}(\alpha) \text { s.t. } 0<l_{J}<\alpha_{1}-\alpha_{2}}(-1)^{|J|+\sum_{i \in I \backslash J} k_{i}}\left((-1)^{k_{1}+k_{2}}-1+(-1)^{k_{2}}-(-1)^{k_{1}}\right) \\
& +\sum_{J \notin \mathcal{T}(\alpha) \text { s.t. } l_{J}<-\left(\alpha_{1}+\alpha_{2}\right)}(-1)^{|J|+\sum_{i \in I \backslash J} k_{i}}\left((-1)^{k_{1}+k_{2}}+1-(-1)^{k_{2}}-(-1)^{k_{1}}\right) \\
& +\sum_{J \notin \mathcal{T}(\alpha) \text { s.t. }-\left(\alpha_{1}+\alpha_{2}\right)<l_{J}<\alpha_{2}-\alpha_{1}}(-1)^{|J|+\sum_{i \in I \backslash J} k_{i}}\left((-1)^{k_{1}+k_{2}}-1-(-1)^{k_{2}}-(-1)^{k_{1}}\right) \\
& \left.+\sum_{J \notin \mathcal{T}(\alpha) \text { s.t. } \alpha_{2}-\alpha_{1}<l_{J}<0}(-1)^{|J|+\sum_{i \in I \backslash J} k_{i}}\left((-1)^{k_{1}+k_{2}}-1+(-1)^{k_{2}}-1\right)\right) .
\end{aligned}
$$

Assuming as in Theorem 1.2 that $k_{m-l}, \ldots, k_{m} \in \mathbb{Z}_{+}$for some integer $l$ and $k_{1}=$ $\cdots=k_{m-l-1}=0$. Then $k_{m-l}+\cdots+k_{m}=m-3$, and we must have $m-l \geq 3$. Thus $k_{1}=k_{2}=0$. Hence, the RHS of (9.2) is equal to

$$
\frac{1}{2} \sum_{J \in \mathcal{T}(\alpha)}(-1)^{|J|+\sum_{i \in I \backslash J} k_{i}}-\frac{1}{2} \sum_{\substack{J \notin \mathcal{T}(\alpha) \text { s.t. } \\-\left(\alpha_{1}+\alpha_{2}\right)<l_{J}<\alpha_{2}-\alpha_{1}<0}}(-1)^{|J|+\sum_{i \in I \backslash J} k_{i}} .
$$

If $J \subset I$ is not triangular and

$$
-\left(\alpha_{1}+\alpha_{2}\right)<l_{J}<\alpha_{2}-\alpha_{1}<0,
$$

then the complement $J^{\prime}:=I \backslash J$ satisfies $\alpha_{1}-\alpha_{2}<l_{J^{\prime}}<\alpha_{1}+\alpha_{2}$ (since $l_{J^{\prime}}=$ $-l_{J}$ ), implying that $J^{\prime}$ is triangular. Conversely, if a subset of $I$ is triangular its complement in $I$ satisfies (9.5). Hence, since $|J|=m-2-\left|J^{\prime}\right|$, the sum (9.4) 
becomes equal to

$$
\begin{aligned}
& \frac{1}{2} \sum_{J \in \mathcal{T}(\alpha)}(-1)^{|J|+\sum_{i \in I \backslash J} k_{i}}-\frac{1}{2} \sum_{J^{\prime} \in \mathcal{T}(\alpha)}(-1)^{m-\left|J^{\prime}\right|+\left(m-3-\sum_{i \in I \backslash J^{\prime}} k_{i}\right)} \\
& =\sum_{J \in \mathcal{T}(\alpha)}(-1)^{m-|J|+\sum_{i \in I \backslash J} k_{i}}
\end{aligned}
$$

which is our formula of Theorem 1.2.

Similarly, if $m$ is odd, the RHS of (9.3) is equal to

$$
\begin{aligned}
& \frac{1}{2}\left(\sum_{J \in \mathcal{T}(\alpha),|J| \text { odd }}(-1)^{\sum_{i \in I \backslash J} k_{i}}\left((-1)^{k_{1}+k_{2}}-1\right)\right. \\
& +\sum_{J \notin \mathcal{T}(\alpha),|J| \text { odd }}(-1)^{\sum_{i \in I \backslash J} k_{i}}\left((-1)^{1+k_{1}+k_{2}}-1\right)+\sum_{J \notin \mathcal{T}(\alpha),|J| \text { odd }}(-1)^{\sum_{i \in I \backslash J} k_{i}}\left((-1)^{k_{1}+k_{2}}-1\right) \\
& l_{J}>\alpha_{1}+\alpha_{2} \quad 0<l_{J}<\alpha_{1}-\alpha_{2} \\
& +\sum_{\substack{J \notin \mathcal{T}(\alpha),|J| \text { odd } \\
l_{J}<-\left(\alpha_{1}+\alpha_{2}\right)}}(-1)^{\sum_{i \in I \backslash J} k_{i}}\left((-1)^{k_{1}+k_{2}}+1\right)+\sum_{\substack{J \notin \mathcal{T}(\alpha),|J| \text { odd } \\
-\left(\alpha_{1}+\alpha_{2}\right)<l_{J}<0}}(-1)^{\sum_{i \in I \backslash J} k_{i}}\left((-1)^{k_{1}+k_{2}}-1\right) \\
& +\sum_{J \in \mathcal{T}(\alpha),|J| \text { even }}(-1)^{\sum_{i \in I \backslash J} k_{i}}\left((-1)^{1+k_{2}}+(-1)^{1+k_{1}}\right) \\
& +\sum_{\substack{J \notin \mathcal{T}(\alpha),|J| \text { even } \\
l_{J}>\alpha_{1}+\alpha_{2}}}(-1)^{\sum_{i \in I \backslash J} k_{i}}\left((-1)^{1+k_{2}}+(-1)^{1+k_{1}}\right) \\
& +\quad \sum(-1)^{\sum_{i \in I \backslash J} k_{i}}\left((-1)^{1+k_{2}}+(-1)^{k_{1}}\right) \\
& \begin{array}{l}
J \notin \mathcal{T}(\alpha),|J| \text { even } \\
0<l_{J}<\alpha_{1}-\alpha_{2}
\end{array} \\
& \left.+\sum_{\substack{\left.J \notin \mathcal{T}(\alpha),|J| \text { even } \\
l_{J}<\alpha_{2}-\alpha_{1}<0\right)}}(-1)^{\sum_{i \in I \backslash J} k_{i}}\left((-1)^{k_{2}}+(-1)^{k_{1}}\right)+\sum_{\substack{J \notin \mathcal{T}(\alpha),|J| \text { odd } \\
\alpha_{2}-\alpha_{1}<l_{J}<0}}(-1)^{\sum_{i \in I \backslash J} k_{i}}\left((-1)^{1+k_{2}}+(-1)^{k_{1}}\right)\right) \\
& =-\sum_{\substack{J \notin \mathcal{T}(\alpha),|J| \text { odd } \\
l_{J}>\alpha_{1}+\alpha_{2}}}(-1)^{\sum_{i \in I \backslash J} k_{i}}+\sum_{\substack{J \notin \mathcal{T}(\alpha),|J| \text { odd } \\
l_{J}<-\left(\alpha_{1}+\alpha_{2}\right)}}(-1)^{\sum_{i \in I \backslash J} k_{i}}-\sum_{J \in \mathcal{T}(\alpha),|J| \text { even }}(-1)^{\sum_{i \in I \backslash J} k_{i}} \\
& -\sum_{\substack{J \notin \mathcal{T}(\alpha),|J| \text { even } \\
l_{J}>\alpha_{1}+\alpha_{2}}}(-1)^{\sum_{i \in I \backslash J} k_{i}}+\sum_{\substack{J \notin \mathcal{T}(\alpha),|J| \text { even } \\
-\left(\alpha_{1}+\alpha_{2}\right)<l_{J}<\alpha_{2}-\alpha_{1}}}(-1)^{\sum_{i \in I \backslash J} k_{i}}+\sum_{\substack{J \notin \mathcal{T}(\alpha),|J| \text { even } \\
l_{J}<-\left(\alpha_{1}+\alpha_{2}\right)}}(-1)^{\sum_{i \in I \backslash J} k_{i}} .
\end{aligned}
$$

If $J \subset I$ is not triangular, $|J|$ is even and $l_{J}<-\left(\alpha_{1}+\alpha_{2}\right)$, then the complement $J^{\prime}:=I \backslash J$ satisfies $l_{J^{\prime}}>\alpha_{1}+\alpha_{2}$, and $\left|J^{\prime}\right|$ is odd and vice versa. Moreover, if $J \subset I$ is not triangular, $|J|$ is even and $-\left(\alpha_{1}+\alpha_{2}\right)<l_{J}<\alpha_{2}-\alpha_{1}<0$, then $J^{\prime}$ is triangular, $\left|J^{\prime}\right|$ is odd and vice versa. Hence, the RHS of (9.3) is equal to

$$
-\sum_{\substack{J \in \mathcal{T}(\alpha) \\|J| \text { even }}}(-1)^{\sum_{i \in I \backslash J} k_{i}}+\sum_{\substack{J \in \mathcal{T}(\alpha) \\|J| \text { odd }}}(-1)^{\sum_{i \in I \backslash J} k_{i}}=\sum_{J \in \mathcal{T}(\alpha)}(-1)^{m-|J|+\sum_{i \in I \backslash J} k_{i}},
$$

which again is our formula from Theorem 1.2

Note that the equivalence of the two formulas then follows from Remark 3.1 which allows us to write any intersection pairing in the form of Theorem 1.2, i.e. 


$$
\int_{\mathcal{M}(\alpha)} c_{m-l}^{k_{m-l}} \cdots c_{m}^{k_{m}}
$$

with $k_{m-l}, \ldots, k_{m} \in \mathbb{Z}_{+}$.

\section{OTHER EXPLICIT FORMULAS FOR INTERSECTION PAIRINGS}

Using the commutativity of geometric quantization and symplectic reduction Takakura obtains in 20 an explicit formula for cohomology intersection pairings on arbitrary polygon spaces. Later Konno obtains an equivalent expression (see [14]), although written in a different basis, using algebro-geometric methods. Their result is the following.

Theorem 10.1 (Konno, Takakura). Let $\alpha=\left(\alpha_{1}, \ldots, \alpha_{m}\right)$ be generic and let $\left(k_{1}, \ldots, k_{m}\right)$ be a sequence of nonnegative integers with $\sum_{i=1}^{m} k_{i}=m-3$. Let $\mathcal{S}(\alpha)$ be the family of short sets for $\alpha$. Then we have

$$
\int_{\mathcal{M}(\alpha)} c_{1}^{k_{1}} \cdots c_{m}^{k_{m}}=-\frac{1}{2} \sum_{R \in \mathcal{S}(\alpha)}(-1)^{|R|+\sum_{i \in R} k_{i}} .
$$

We will now see how this formula is equivalent to Theorem 1.2 although our formula uses a smaller family of sets (the triangular sets $\mathcal{T}(\alpha)$ ) which is always contained in $\mathcal{S}(\alpha)$.

Proof. Let us again assume without loss of generality that $\alpha_{1}>\alpha_{2}$ and let $I=$ $\{3, \ldots, m\}$. Notice that

$$
\begin{aligned}
& \mathcal{S}(\alpha)=\{R \subset I \mid R \in \mathcal{T}(\alpha) \cap \mathcal{S}(\alpha)\} \cup\{R \subset I \mid R \notin \mathcal{T}(\alpha) \text { and } R \in \mathcal{S}(\alpha)\} \\
& \cup\{R=\{1,2\} \cup J \mid J \subset I \text { and } R \in \mathcal{S}(\alpha)\} \cup\{R=\{1\} \cup J \mid J \subset I \text { and } R \in \mathcal{S}(\alpha)\} \\
& \cup\{R=\{2\} \cup J \mid J \subset I \text { and } R \in \mathcal{S}(\alpha)\} .
\end{aligned}
$$

Again let $S_{R}$ and $l_{J}$ be respectively equal to

$$
S_{R}:=\sum_{i \in R} \alpha_{i}-\sum_{i \notin R} \alpha_{i} \text { and } l_{J}:=\sum_{i=3}^{m}(-1)^{\chi_{I \backslash J}(i)} \alpha_{i},
$$

whenever $J \subset I$. Note that the elements of $\mathcal{S}(\alpha)$ are precisely the subsets of $\{1, \ldots, m\}$ for which $S_{R}<0$.

As we saw before in the proof of Theorem 9.4, if $R \subset I$ and $R \in \mathcal{T}(\alpha)$, then $S_{R}<0$, and so $R \in \mathcal{S}(\alpha)$. On the other hand, if $R \subset I$ is not triangular, then $R \in \mathcal{S}(\alpha)$ if and only if $l_{R} \leq 0$ or $0<l_{R}<\alpha_{1}-\alpha_{2}$.

If $R=\{1,2\} \cup J$ with $J \subset I$, we have $|R|=|J|+2$ and $S_{R}=l_{J}+\alpha_{1}+\alpha_{2}$, and then $R \in \mathcal{S}(\alpha)$ if and only if $J \notin \mathcal{T}(\alpha)$ and $l_{J}<-\left(\alpha_{1}+\alpha_{2}\right)$.

If $R=\{1\} \cup J$ with $J \subset I$, then $|R|=|J|+1$ and $S_{R}=l_{J}+\alpha_{1}-\alpha_{2}$, and so $R \in \mathcal{S}(\alpha)$ exactly when $J \notin \mathcal{T}(\alpha)$ and $l_{J}<-\left(\alpha_{1}-\alpha_{2}\right)$.

Similarly, if $R=\{2\} \cup J$ with $J \subset I$, we have $|R|=|J|+1$ and $S_{R}=l_{J}+\alpha_{2}-\alpha_{1}$, and so $R \in \mathcal{S}(\alpha)$ if and only if $J \notin \mathcal{T}(\alpha)$ and $l_{J}<\alpha_{1}+\alpha_{2}$. 
Putting this information together, the RHS of (10.1) is equal to

$$
\begin{aligned}
& -\frac{1}{2}\left(\sum_{J \in \mathcal{T}(\alpha)}(-1)^{|J|+\sum_{i \in J} k_{i}}+\sum_{J \notin \mathcal{T}(\alpha)} \sum_{\substack{\text { s.t. } \\
l_{J}<\alpha_{1}-\alpha_{2}}}(-1)^{|J|+\sum_{i \in J} k_{i}}+\sum_{J \notin \mathcal{T}(\alpha)} \sum_{\text {s.t. } l_{J}<-\left(\alpha_{1}+\alpha_{2}\right)}(-1)^{|J|+\sum_{i \in J} k_{i}}\right. \\
& \left.-\sum_{J \notin \mathcal{T}(\alpha) \text { s.t. } l_{J}<-\left(\alpha_{1}-\alpha_{2}\right)}(-1)^{|J|+\sum_{i \in J} k_{i}}-\sum_{J \notin \mathcal{T}(\alpha)} \sum_{\text {s.t. } l_{J}<\alpha_{1}+\alpha_{2}}(-1)^{|J|+\sum_{i \in J} k_{i}}\right) \\
& =-\frac{1}{2}\left(\sum_{J \in \mathcal{T}(\alpha)}(-1)^{|J|+\sum_{i \in J} k_{i}}-\sum_{\substack{J \notin \mathcal{T}(\alpha) \text { s.t. } \\
\left(\alpha_{1}+\alpha_{2}\right)<l_{J}<\alpha_{2}-\alpha_{1}<0}}(-1)^{|J|+\sum_{i \in J} k_{i}}\right) .
\end{aligned}
$$

As we saw before, if $J \subset I$ is not triangular and

$$
-\left(\alpha_{1}+\alpha_{2}\right)<l_{J}<\alpha_{2}-\alpha_{1}<0,
$$

then the complement $J^{\prime}:=I \backslash J$ satisfies $\alpha_{1}-\alpha_{2}<l_{J^{\prime}}<\alpha_{1}+\alpha_{2}$, implying that $J^{\prime}$ is triangular. Conversely, if a subset of $I$ is triangular, its complement in $I$ satisfies (10.2). Hence, since $|J|=m-2-\left|J^{\prime}\right|$ and $\sum_{i \in I \backslash J} k_{i}=(m-3)-\sum_{i \in J} k_{i}$, the RHS of (10.1) becomes equal to

$$
\begin{aligned}
& \frac{1}{2}\left(\sum_{J \in \mathcal{T}(\alpha)}(-1)^{|J|+\sum_{i \in I \backslash J} k_{i}+m}+\sum_{J^{\prime} \in \mathcal{T}(\alpha)}(-1)^{m-\left|J^{\prime}\right|+\sum_{i \in I \backslash J^{\prime}} k_{i}}\right) \\
& =\sum_{J \in \mathcal{T}(\alpha)}(-1)^{m-|J|+\sum_{i \in I \backslash J} k_{i}},
\end{aligned}
$$

which is our formula of Theorem 1.2 ,

Again the equivalence of the two formulas then follows from Remark 3.1. which allows us to write any intersection pairing in the form of Theorem 1.2 .

$$
\int_{\mathcal{M}(\alpha)} c_{m-l}^{k_{m-l}} \cdots c_{m}^{k_{m}}, \quad k_{m-l}, \ldots, k_{m} \in \mathbb{Z}_{+}
$$

\section{ACKNOWLEDGEMENTS}

We are grateful to Jonathan Weitsman for suggesting this problem and for many useful conversations and remarks. We would also like to thank Jean-Claude Hausmann and the referee for their comments on earlier versions of this work and Tatsuru Takakura and Hiroshi Konno for bringing their work to our attention.

\section{REFERENCES}

1. M. Brion, Cohomologie équivariante des points semi-stables, J. Reine Angew. Math. 421 (1991), 125-140. MR:1129578 (92i:14010)

2. J. J. Duistermaat and G. J. Heckman, On the variation in the cohomology of the symplectic form of the reduced phase-space, Invent. Math. 69 (1982), 259-268. MR674406 (84h:58051a)

3. V. Guillemin, Moment Maps and Combinatorial Invariants of Hamiltonian $T^{n}$-spaces, Progress in Mathematics, 122, Birkhäuser, Boston, 1994. MR1301331(96e:58064)

4. R. Graham, D. Knuth and O. Patashnik, Concrete mathematics. A foundation for computer science (2nd edition), Addison-Wesley Publishing Company, Reading, MA, 1994. MR1397498 (97d:68003) 
5. V. Guillemin and S. Sternberg, Geometric quantization and multiplicities of group representations, Invent. Math. 67 (1982), 515-538. MR664118 (83m:58040)

6. J.-C. Hausmann, Sur la topologie des bras articulés, Algebraic Topology Poznan 1989, 146159, Lecture Notes in Math., 1474, Springer, Berlin, 1991. MR1133898 (93a:57035)

7. J.-C. Hausmann and A. Knutson, Polygon spaces and Grassmannians, Enseign. Math. (2) 43 (1997), 173-198. MR 1460127 (98e:58035)

8. J.-C. Hausmann and A. Knutson, The cohomology ring of polygon spaces, Ann. Inst. Fourier (Grenoble) 48 (1998), 281-321. MR1614965 (99a:58027)

9. L. Jeffrey, Extended moduli spaces of flat connections on Riemann surfaces, Math. Ann. 298 (1994), 667-692. MR:1268599 (95g:58030)

10. L. Jeffrey and J. Weitsman, Toric structures on the moduli space of flat connections on a Riemann surface. II. Inductive decomposition of the moduli space, Math. Ann. 307 (1997), 93-108. MR1427677 (97m:58027)

11. Y. Karshon, Periodic Hamiltonian flows on four-dimensional manifolds, Mem. Amer. Math. Soc. 141 (1999). MR1612833(2000c:53113)

12. F. Kirwan, The cohomology rings of moduli spaces of bundles over Riemann surfaces, J. Amer. Math. Soc. 5 (1992), 853-906. MR1145826 (93g:14016)

13. A. Klyachko, Spatial polygons and stable configurations of points in the projective line, Algebraic geometry and its applications (Yaroslavl', 1992), 67-84, Aspects Math. E25, Vieweg, Braunschweig, 1994. MR1282021 (95k:14015)

14. H. Konno, The intersection pairings on the configuration spaces of points in the projective line, J. Math. Kyoto Univ. 41 (2001), 277-284. MR.1852984(2002g:14081)

15. M. Kontsevich, Intersection theory on the moduli space of curves and the matrix Airy function, Comm. Math. Phys. 147 (1992), 1-23. MR.1171758 (93e:32027)

16. M. Kapovich and J. Millson, The symplectic geometry of polygons in Euclidean space, J. Differential Geom. 44 (1996), 479-513. MR.1431002 (98a:58027)

17. Y. Kamiyama and M. Tezuka, Symplectic volume of the moduli space of spatial polygons, J. Math. Kyoto Univ. 39 (1999), 557-575. MR1718781 (2000g:53096)

18. E. Lerman, Symplectic cuts, Math. Res. Lett. 2 (1995), 247-258. MR1338784 (96f:58062)

19. A. Mandini, The geometry of the moduli space of polygons in the Euclidean space, Ph.D. Thesis, Università di Bologna, 2007.

20. T. Takakura, Intersection theory on symplectic quotients of products of spheres, Internat. J. Math. 12 (2001), 97-111. MR1812066 (2002k:53165)

21. $\mathrm{Vu}$ The Khoi, On the symplectic volume of the moduli space of spherical and Euclidean polygons, Kodai Math. J. 28 (2005), 199-208. MR2122200(2005i:53112)

22. K. Walker, Configuration spaces of linkages, Undergraduate Thesis, Princeton (1985).

23. J. Weitsman, Geometry of the intersection ring of the moduli space of flat connections and the conjectures of Newstead and Witten, Topology 37 (1998), 115-132. MR 1480881 (99m:57030)

24. E. Witten, Two-dimensional gravity and intersection theory on moduli space, Surveys in differential geometry (Cambridge, MA, 1990), 243-310, Lehigh Univ., Bethlehem, PA, 1991. MR 1144529 (93e:32028)

25. E. Witten, On quantum gauge theories in two dimensions, Comm. Math. Phys. 141 (1991), 153-209. MR1133264 (93i:58164)

26. E. Witten, On the Kontsevich model and other models of two-dimensional gravity, Proceedings of the XXth International Conference on Differential Geometric Methods in Theoretical Physics, Vol. 1, 2 (New York, 1991), 176-216, World Sci. Publ., River Edge, NJ, 1992. MR:1225112 (94g:32029)

27. T. Yoshida, The generating function for certain cohomology intersection pairings of the moduli space of flat connections, J. Math. Sci. Univ. Tokyo 8 (2001), 541-558. MR 1855458 (2002g:53152)

Departamento De Matemática, Instituto Superior Técnico, Av. Rovisco Pais, 1049001 Lisbon, Portugal

E-mail address: agapito@math.ist.utl.pt

Departamento De Matemática, Instituto Superior Técnico, Av. Rovisco Pais, 1049001 Lisbon, Portugal

E-mail address: lgodin@math.ist.utl.pt 